\title{
Feasibility of skin-friction field measurements in a transonic wind tunnel using a global luminescent oil film
}

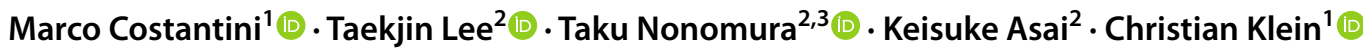

Received: 15 May 2020 / Revised: 20 November 2020 / Accepted: 1 December 2020 / Published online: 8 January 2021

(c) The Author(s) 2021

\begin{abstract}
The feasibility of skin-friction field measurements using the global luminescent oil-film skin-friction field estimation method was evaluated for a challenging case of a supercritical airfoil model under transonic wind-tunnel conditions (freestream Mach number of 0.72) at a high Reynolds number (10 million, based on the model chord length). The oil-film thickness and skin-friction coefficient distributions were estimated over the airfoil model upper surface for a range of angles of attack (from $-0.4^{\circ}$ to $2.0^{\circ}$ ), thus enabling the study of different boundary-layer stability situations with laminar-turbulent transition, including cases with shock-wave/boundary-layer interaction. Conventional pressure measurements on the surface and in the wake of the model as well as Schlieren flow visualizations were conducted to support the oil-film based investigations. In the laminar-flow regions, the oil-film thickness could be generally kept below the critical limit of roughness that would induce premature boundary-layer transition. The skin friction in this region could be estimated with a moderate confidence level, as confirmed for portions of the chord by the reasonable agreement with numerical data obtained via laminar boundary-layer computations. Moreover, the location of transition onset was evaluated from the skin-friction estimations with relatively low uncertainty, thus enabling the examination of the transition location evolution with varying angle of attack. The estimated locations of transition onset were shown to be in general agreement with reference transition locations measured via temperature-sensitive paint. On the other hand, the oil-film thickness in the turbulent-flow regions was larger than the height of the viscous sublayer, which led to an hydraulically rough surface with increased skin friction, as compared to the clean configuration. For this reason, quantitative skin-friction estimations were not feasible in the turbulent-flow regions. The global effects of the oil-film setup on the flow around the airfoil were evaluated from the estimations of the aerodynamic coefficients. In particular, it was shown that the presence of the specific base coat used for the application of the oil film already induced a significant increase in airfoil drag, as compared to the clean configuration, whereas a thin oil film led to negligible or small additional increases in drag. Based on the present observations, considerations for the further improvement of the global luminescent oil-film skin-friction field estimation method in transonic flow experiments at high Reynolds numbers are elucidated.
\end{abstract}

Marco Costantini

marco.costantini@dlr.de

1 Institute of Aerodynamics and Flow Technology, German Aerospace Center (DLR), Bunsenstr. 10, Göttingen 37073, Germany

2 Department of Aerospace Engineering, Tohoku University, Aoba-Ku, Sendai, Miyagi 980-8579, Japan

3 PRESTO, JST, Aoba-Ku, Sendai, Miyagi 980-8579, Japan 


\section{Graphic abstract}
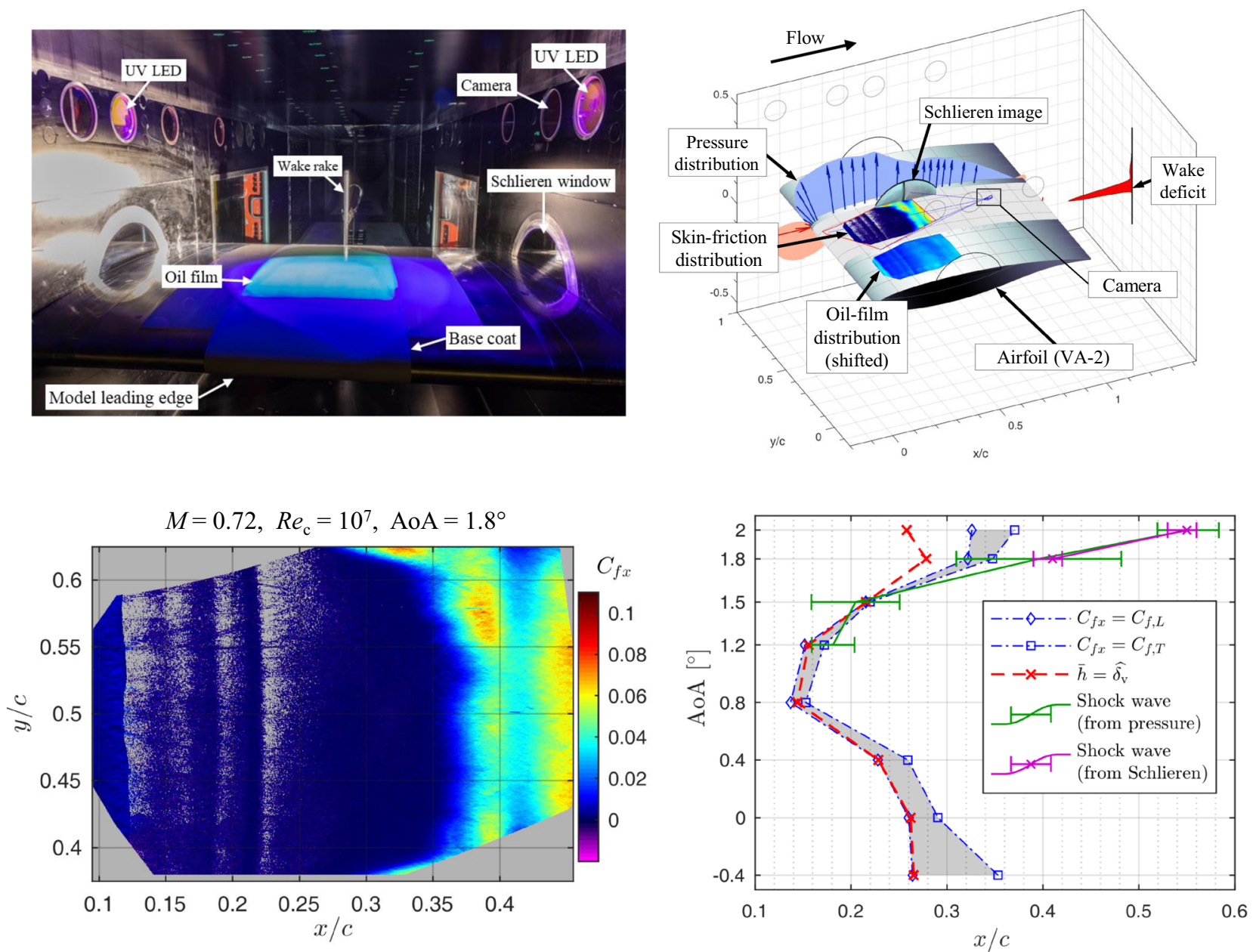

\section{Introduction}

Skin friction is deeply related to boundary-layer characteristics, laminar-turbulent transition, and vortical structures, and its measurement is required for the validation of the computational fluid dynamics (CFD) approach. In particular, its knowledge is crucial for understanding the characteristics of the shock-wave/boundary-layer interaction, which is an important flow phenomenon that must be predicted and controlled for stable, low-drag cruise in the transonic flow regime (Bohning and Zierep 1986; Thiede et al. 1984). However, the transonic, high Reynolds number flow regime is challenging for the experimental measurement of the skin-friction field. Especially at these flow conditions, surface roughness is a key factor in the aforementioned flow phenomena. Therefore, measurement sensors should not add surface roughness, or the surface roughness should be controlled. In practical challenges, modifying or manufacturing special models for skin-friction measurement may be limited due to the compatibility of other measurement techniques and to the wind-tunnel test schedule (if the model should be modified or exchanged). Depending on the model shape, the installation of shear stress sensors may be impossible or impractical. Therefore, optical measurement approaches are appropriate for the production of a suitable amount of data within practical conditions.

The most common skin-friction measurement technique using an optical system is the oil-film interferometry (OFI), proposed by Tanner and Blows (1976). This technique measures the deformation of an oil film using interferometry, and the skin friction is calculated from the spatiotemporal interferometry data. Examples of applications of OFI in wind-tunnel experiments on airfoil models are reported in Monson et al. (1993) and Driver (2003), among 
others, while Naughton and Liu (2007) considered photogrammetric effects of the camera position to an object. OFI measurements require additional information on the flow direction for the determination of the skin friction. Naughton and Brown (1996) used fluorescent tracers to determine the flow direction, whereas Lunte and Schülein (2020) proposed an algorithm to calculate the skin-friction field automatically. The particle image surface flow visualization (PISFV) method (Mosharov et al. 2006, 2011; Husen et al. 2015) uses cross-correlation analysis for the oil-flow vector determination. The global luminescent oil film (GLOF) is another oil-film-based technique developed by Liu et al. (2008). This technique measures an oil-film thickness by the luminescent intensity of the oil film. In its original formulation, an optical-flow method was proposed to extract the skinfriction field from the oil-film images. Using GLOF, relative (normalized) skin-friction distributions were obtained for low-speed flows in Woodiga and Liu (2009); Liu et al. (2011); Husen et al. (2014); Zhong et al. (2015); and Liu (2019). A further GLOF image analysis method based on the linear least-squares (LLS) method was presented in Lee et al. (2018), where it was applied to extract relative skinfriction fields in subsonic and supersonic flows. The LLSbased method was validated for quantitative skin-friction measurements through an experiment on a flat-plate model in a low-speed flow (Lee et al. 2020c). This method has been also extended to obtain relative skin-friction distributions in a low-speed, unsteady flow field (Lee et al. 2020a, b).

In recent years, an advanced approach using heat-flux data to extract the skin-friction field, in which surface temperature data measured by temperature-sensitive paint (TSP) are analyzed, has been proposed (Liu and Woodiga 2011; Liu 2019). Using this approach, skin-friction fields were obtained from TSP data in underwater applications (Miozzi et al. 2019, 2020). The application of the TSP-based approach for transonic air flows is still on-going and requires validation.

The VicToria project, an internal research project of the German Aerospace Center (DLR), aimed to build digital integrated tools for aircraft development, which would encourage the adaptation of new technologies while at the same time avoiding technological risks. ${ }^{1}$ One of the goals of the DLR VicToria project was the development and quantification of measurement techniques for the physical modeling and validation of numerical tools. Validation of CFD is a key factor for the successful development of digital tools, and the skin-friction field is one of the most important evaluation parameters for the validation. In the present study, the feasibility of the GLOF measurement technique

\footnotetext{
1 https://www.dlr.de/as/en/desktopdefault.aspx/tabid-11460/20078 _read-47033/.
}

to experimentally obtain skin-friction fields was examined for transonic flow conditions (freestream Mach number of 0.72 ) at a high chord Reynolds number of $10^{7}$. Schlieren flow visualizations and conventional pressure measurements (on the model and in its wake) were performed for cross-validation. The experiments were conducted in the Transonic Wind Tunnel Göttingen of the German-Dutch Wind Tunnels (DNW-TWG), and the reference model was the supercritical airfoil model VA-2, which was designed for a small change in the shock-wave location at off-design conditions and has been used in various studies (Thiede and Krogmann 1989; Rubesin and Viegas 1989; Mateer et al. 1992; Krogmann et al. 1984; Krenkel 2012; Leuckert 2012). It should be emphasized here that most of these studies focused on turbulent boundary layers, except for Leuckert (2012), where free transition was examined; however, the investigated range of Mach numbers was below $M=0.72$, as considered in the present work. Reference transition locations have become available only recently (Costantini et al. 2020) via TSP measurements conducted for the same test cases as those examined in this work.

In this study, the GLOF measurement technique was applied to the airfoil model upper surface at transonic flow conditions for various boundary-layer stability situations, implemented via a variation of the model angle of attack from $-0.4^{\circ}$ to $2.0^{\circ}$. Laminar-turbulent transition was observed on the investigated surface region for all examined test conditions; at the larger angles of attack, shockwave/boundary-layer interaction was also seen on the model surface.

The structure of the present work can be summarized as follows. First, the theoretical background for the extraction of skin-friction fields from luminescent oil images, including the requirements on the measurement conditions, is presented in Sect. 2. The experimental setup and the examined test conditions are described in Sect. 3. An a priori estimation of the measurement conditions required for the present experiments is discussed in Sect. 4; in that section, also the calibration procedure (necessary for the estimation of the skin-friction field from GLOF image data) and the wind-tunnel operation sequence are described. The extraction process of the oil-film thickness and skin-friction distributions from the luminescent oil images, including the camera calibration and image projection procedures, is discussed in Sect. 5, where an estimation of the measurement uncertainties is also reported. The experimental results are presented and analyzed in Sect. 6, with emphasis on the accuracy of the quantitative skin-friction estimations in the laminar-flow regions, as well as on the issues limiting the repeatability of the results and the capability to obtain quantitative skin-friction fields in the turbulent-flow regions. The findings of the present study are summarized in Sect. 7, 
where recommendations for future GLOF measurements in transonic, high Reynolds number flows are also presented.

\section{Theoretical background}

\subsection{Global luminescent oil-film skin-friction field estimation}

The Global Luminescent Oil-Film Skin-Friction field Estimation method (GLOFSFE) is an image-based method that measures the spatio-temporal development of the thickness of an oil film based on its luminescent intensity; processing the GLOF data enables the attainment of the skin-friction field (Liu et al. 2008; Lee et al. 2018). In the present work, only general information is reported about this measurement technique and the related data analysis. For a detailed explanation, discussion, and validation in a low-speed flow, the reader is referred to earlier work, and in particular to Liu et al. (2008); Lee et al. (2018) and Lee et al. (2020c).

In wind-tunnel experiments, the development of a thin oil film applied to the surface of a wind-tunnel model depends, in general, on the wall shear stress, on the pressure gradient, and on the body force. The governing equation of the oil film, i.e., the thin-oil-film equation (Brown and Naughton 1999), is:

$\frac{\partial h}{\partial t}+\nabla \cdot\left(\frac{h^{2}}{2 \mu} \boldsymbol{\tau}-\frac{h^{3}}{3 \mu}(\nabla p-\rho \mathbf{a})\right)=0$,

where $h$ the oil-film thickness, $t$ is the time, $\tau$ is the skinfriction vector, $\mu$ is the oil dynamic viscosity, $p$ is the pressure, $\rho$ is the oil density, and $\mathbf{a}$ is the body acceleration.

In the case of a luminescent oil film, the distribution of the luminescent intensity emitted from the oil is typically recorded by means of a camera system. When the skin friction exerts its action on the interface between the gas (usually air) and the oil, the oil-film thickness varies according to Eq. (1). The measured luminescent intensity $I$, emitted from an optically thin oil film, is proportional to the oil-film thickness and to the excitation light intensity (Liu and Sullivan 1998; Husen et al. 2018), i.e.:

$I=I_{\mathrm{ref}} \frac{h}{h_{*}}$,

where $I_{\text {ref }}$ is the reference intensity value, which essentially corresponds to the excitation light intensity distribution (see Sects. 2.2 and 4.2); and $h_{*}$ is the unit oil-film thickness, which is a calibration coefficient. The calibration information is obtained by following Lee et al. (2020c); the calibration procedure will be described in Sect. 4.2.
In the present study, the LLS-based method (Lee et al. 2018,2020 c) was used for the GLOF image analysis. As discussed in detail in those publications, this method enables the extraction of the skin-friction field from a sequence of GLOF images with a sufficient number of oil-film image pairs. In this case, the $\boldsymbol{\tau}$-field is assumed to be time-independent, and the optimal skin-friction field that satisfies Eq. (1) for the given data is determined. In practice, a finite-difference form of Eq. (1) is considered, which can be rearranged into an overdetermined system of equations for an LLS solution. The estimated skin friction $\hat{\tau}_{i}$ in the $i$-direction $(i=x, y$, where $x$ and $y$ are the stream-wise and span-wise directions, respectively) is obtained as:

$\hat{\tau}_{i}=\tau_{*} \hat{\tilde{\tau}}_{i}=\frac{\mu x_{*}}{t_{*} h_{*}} \hat{\tilde{\tau}}_{i}$,

where $\hat{\tilde{\tau}}_{i}$ is the estimated normalized skin friction in the $i$-direction (obtained from the GLOF image analysis), $\tau_{*}$ the unit skin friction, $\mu$ the oil dynamic viscosity, $x_{*}$ the unit length of the spatial resolution, $t_{*}$ the time interval of the image pair, and $h_{*}$ the aforementioned unit oil-film thickness. Note that, for simplicity, a single $x_{*}$ has been used in Eq. (3), but two different unit lengths can be considered for the spatial resolution in the stream-wise and span-wise directions. For a time sequence of GLOF images, the LLS method provides the estimation of the time-averaged, normalized skin-friction field $\hat{\tilde{\tau}}$. In the numerical procedure, the image pairs and the skin-friction field are vectorized, i.e., matrices are converted into column vectors. In particular, $\hat{\tilde{\boldsymbol{\tau}}}$ is vectorized as a high-dimensional vector $\underline{\tilde{\tilde{\boldsymbol{\tau}}}}$. Moreover, the GLOF images are projected onto a structured grid representing the surface of interest. The residual defined from the physical model (i.e., Eq. (1)) is then expressed as a linear system of equations. In the following equations, the effects of the pressure gradient and of the body force terms on the oil-film thickness development will be assumed to be negligible, as compared to the effect of the skin-friction term. As will be discussed in Subsection 2.3.3, this assumption is not mandatory for the application of the GLOFSFE, but it allows a simplification of the present description. In this case, the LLS method provides $\underline{\tilde{\tilde{\tau}}}$ as (Björck 1996; Lee et al. 2018, 2020c):

$\hat{\tilde{\tau}}=\mathbf{C}^{-1} \mathbf{d}$

where

$\mathbf{C}=\frac{1}{4} \sum_{k}\left\{\operatorname{diag}\left(\mathbf{M}_{\mathrm{c} 2 f_{-k}}\right)\right\}^{2} \boldsymbol{\Delta}_{\mathbf{x}}^{\mathrm{T}} \boldsymbol{\Delta}_{\mathbf{x}}\left\{\operatorname{diag}\left(\mathbf{M}_{\mathrm{c} 2 f_{-}} \boldsymbol{r}_{k}\right)\right\}^{2}$,

and 
$\mathbf{d}=-\frac{1}{2} \sum_{k}\left\{\operatorname{diag}\left(\mathbf{M}_{\mathrm{c} 2 \mathrm{r}} \boldsymbol{r}_{-k}\right)\right\}^{2} \boldsymbol{\Delta}_{\mathbf{x}}^{\mathrm{T}} \boldsymbol{\Delta}_{\mathbf{t}} \boldsymbol{r}_{-k}$.

The operators, vectors, matrices, and dimensions used in Eqs. (5) and (6) are defined as follows: 'diag' indicates the diagonal matrix operator; $\boldsymbol{r}_{k}$ is the vectorized ratioed image $\left(I / I_{\text {ref }}\right)$ pair at the moment $k$, where the intensity ratio is computed for each pixel prior to the projection of the ratioed images onto the surface grid; $\boldsymbol{\Delta}_{x} \in \mathfrak{R}^{M \times N}$ is the spatial difference scheme matrix; $\boldsymbol{\Delta}_{t} \in \mathfrak{R}^{M \times 2 M}$ is the temporal difference scheme matrix; $\boldsymbol{M}_{\mathbf{c 2 f}} \in \mathfrak{R}^{N \times P}$ is the cell-to-face interpolation matrix; $N$ is the dimension of the vector $\hat{\tilde{\boldsymbol{\tau}}} ; P$ is the dimension of the the vector $\underline{r}_{k}$; and $M$ is the total number of the nodes of the surface grid.

The coefficient of determination $R^{2}$, as defined by Lee et al. (2018), can be used to assess the quality of the estimated $\hat{\tilde{\tau}}$ : in fact, $R^{2}=1$ would indicate that all data are perfectly represented by the LLS regression, whereas a small value of $R^{2}$ would indicate a poor regression. Indications on the normalized root-mean-square error (NRMSE) in the estimated skin friction are reported in Lee et al. (2018) for various values of $R^{2}$, which were obtained with various combinations of image parameters (such as the image noise level) in simulated GLOF images. Values of $R^{2}$ above 0.5 indicatively corresponded to NRMSE values below $\pm 5 \%$

The calibration parameters $\mu, x_{*}$, and $t_{*}$ in Eq. 3 are given by the used oil, by the spatial resolution of the camera system, and by the time interval between the GLOF images, respectively. The calibration parameter $h_{*}$ is determined according to the ratioed image film thickness method (Husen et al. 2018), which is based on the oil-droplet volume method. In practice, the unit thickness $h_{*}$ is estimated using measurement results according to the following equation:

$h_{*}=\frac{\bar{h}}{\overline{r_{\text {cal }}}}=\frac{v_{\text {droplet }} / S}{\overline{r_{\text {cal }}}}=\frac{v_{\text {droplet }}}{n_{\text {cal }} \overline{r_{\text {cal }}} x_{*}^{2}}$,

where $\bar{i}$ indicates the ensemble average of an arbitrary quantity $i, \overline{r_{\text {cal }}}$ is the ensemble-averaged ratioed image of the droplet, $S$ is the corresponding area, and $n_{\text {cal }}$ is the total pixel number of the area.

\subsection{Photogrammetry and determination of the oil-film thickness}

Measuring the three-dimensional model surface by a twodimensional camera sensor involves the perspective projection transformation. Photogrammetric techniques can be used in wind-tunnel tests to obtain quantitative flow-visualization image data mapped onto the model surface, as done, for example, in Liu et al. (2000).

The three-dimensional coordinates $(x, y, z)$ in the object space and the corresponding two-dimensional coordinates in the image plane $(X, Y)$ are given by the collinearity equations (Mikhail et al. 2001):

$$
\begin{aligned}
X & -X_{p}+d X \\
& =-C \frac{m_{11}\left(x-x_{C}\right)+m_{12}\left(y-y_{C}\right)+m_{13}\left(z-z_{C}\right)}{m_{31}\left(x-x_{C}\right)+m_{32}\left(y-y_{C}\right)+m_{33}\left(z-z_{C}\right)}, \\
Y & -Y_{p}+d Y \\
& =-C \frac{m_{21}\left(x-x_{C}\right)+m_{22}\left(y-y_{C}\right)+m_{23}\left(z-z_{C}\right)}{m_{31}\left(x-x_{C}\right)+m_{32}\left(y-y_{C}\right)+m_{33}\left(z-z_{C}\right)},
\end{aligned}
$$

where $\left(X_{p}, Y_{p}\right)$ is the principal point, $(d X, d Y)$ is the shift of the image point due to the lens distortion, $C$ is the camera principal distance, $\left(x_{C}, y_{C}, z_{C}\right)$ is the camera location in the object space, and $m_{i j}(i, j=1,2,3)$ are the elements of the rotational matrix, which are functions of the Euler orientation angle $(\omega, \phi, \kappa)$. The orientation angles $(\omega, \phi, \kappa)$ are the pitch, yaw, and roll angles, respectively, of the camera in the object coordinate system. The lens distortions $(d X, d Y)$ include the radial and tangential distortions.

Similar to OFI measurements, the surface angle to the camera position should be considered for the measurement of the oil-film thickness at each location on the surface (Naughton and Liu 2007). The oil-film thickness normal to the model surface is (Husen et al. 2018; Lee et al. 2020c):

$h=h_{*} \frac{I-I_{\mathrm{dark}}}{I_{\mathrm{exc}}-I_{\mathrm{dark}}} \cos \theta_{P}$,

where $I$ is the image intensity, $I_{\text {dark }}$ is the dark current image (which accounts for the influence of residual light and camera electronic noise), and $I_{\text {exc }}$ is the excitation light distribution image. The angle $\theta_{P}$ between the surface and the camera position is calculated as:

$\cos \theta_{P}=\frac{\mathbf{n} \cdot \mathbf{v}_{\text {camera }}}{|\mathbf{n}|\left|\mathbf{v}_{\text {camera }}\right|}$,

where $\mathbf{n}$ is the normal vector to the model surface, and $\mathbf{v}_{\text {camera }}$ is the vector from the model surface point to the optical center. It should be noted here that, according to Husen et al. (2018), the resolution of the oil-film thickness increases when $\theta_{P}$ increases, provided that the signal-to-noise ratio of the image corresponding to the quotient of the emission and excitation intensity distributions is the same.

\subsection{Requirements on the measurement conditions}

Since the GLOFSFE is based on assumptions, requirements must be fulfilled for quantitative skin-friction measurements. These can be categorized into fluid dynamic and analytical requirements. In this and in the following sections, the skin-friction vector field will be assumed to be oriented in the stream-wise direction, i.e., $\tau=\tau_{x}$, since the freestream 
examined in the present work was quasi-two-dimensional. It should be, however, emphasized that both components of the skin-friction vector $\left(\tau_{x}\right.$ and $\left.\tau_{y}\right)$ were obtained using the GLOFSFE. In fact, the results presented in Sect. 6 will show the stream-wise component of the skin-friction coefficient $C_{f x}$.

\subsubsection{Critical limit of roughness}

The requirement dictated by the fluid dynamics is whether the oil film affects the flow field, since the oil film adds surface roughness by its shape. The roughness should be kept below a certain critical level. In the laminar-flow regions, a roughness Reynolds number $R e_{k}$ satisfying:

$R e_{k}=\frac{u_{k} k}{v_{k}}>R e_{k, \mathrm{cr}}$

would induce premature laminar-turbulent transition (Braslow 1966). In Eq. (11), $u_{k}$ is the stream-wise velocity component at the top of roughness, $k$ is the roughness height, $v_{k}$ is the local kinematic viscosity of the gas at the top of the roughness, and $R e_{k, \mathrm{cr}}$ is the critical roughness Reynolds number. The values with the subscript " $k$ " are evaluated at the wall-normal location $z=k$ but in the absence of the roughness, i.e., for the equivalent smooth-wall laminar boundary layer. Braslow et al. (1966) determined a general number of $R e_{k, \mathrm{cr}}=600$ for distributed roughness, and Smith and Clutter (1959) reported $R e_{k, \text { cr }}=125$ for two-dimensional roughness. At this point, it should be emphasized that these critical values should be regarded as indicative. In fact, critical roughness Reynolds numbers as low as $R e_{k, \mathrm{cr}} \sim 40-50$ were also found for two-dimensional roughness (Smith and Clutter 1959). Especially, the roughness shape and position as well as the boundary-layer stability situation may have an influence on the transition sensitivity with respect to the roughness effect (Smith and Clutter 1959; Costantini 2016). In the case of two-dimensional bumps, the critical roughness height has been mainly related to the ratio between the bump height and the bump length (Holmes et al. 1985), but factors such as the pressure gradient and the Mach number may also have an effect on the bump influence on boundary-layer stability and transition (Costantini et al. 2019). For threedimensional roughness elements, Braslow (1966) reported $R e_{k, \mathrm{cr}}=100-900$ (depending on the ratio between wallnormal and span-wise size of the roughness element). As a prerequisite for the natural laminar-turbulent transition, the oil-film thickness $h$ should be lower than the critical roughness height $k$.

In the turbulent-flow regions, the oil-film surface should be hydraulically smooth to prevent an increase in the skin friction, as compared to the clean (oil-free) surface. As discussed in Lee et al. (2020c), the oil-film surface roughness is a function of $h$, and the condition of hydraulic smoothness is provided when the time-averaged oil-film thickness $h$ is lower than the viscous sublayer thickness, i.e.:

$h<\delta_{\mathrm{v}}$,

where $\delta_{\mathrm{v}}=5 v_{\mathrm{gas}} / u_{\tau}$ is the viscous sublayer thickness, and $u_{\tau}=\sqrt{\tau / \rho_{\text {gas }}}$ is the friction velocity.

\subsubsection{Courant-Friedrichs-Lewy condition}

A first analytical requirement is related to the approximation of the partial derivatives of the relevant variables via the finite-difference method (see Eqs. (3)-(6)). The Courant-Friedrichs-Lewy (CFL) condition (Courant et al. 1967) can be defined as:

$C F L=\left.\tilde{u}\right|_{z=h}=r \tilde{\tau}=\frac{\tau h}{\mu} \frac{t_{*}}{x_{*}}<C F L_{\max }$,

where $C F L$ is the CFL number, $\left.\tilde{u}\right|_{z=h}$ is the normalized velocity (pixels per frame) at the oil surface, and $C F L_{\max }$ is the maximally allowable CFL number. In the GLOFSFE, the governing equation is solved only once per given image pair and the error is not accumulated. Therefore, the maximally allowable CFL number can be larger than one. In fact, the partial derivatives can be valid depending on the scheme and the given data, so that values of $C F L$ moderately larger than one can be allowed (see Sect. 6.1).

\subsubsection{Neglection of pressure gradient and body force effects}

A second analytical condition is the assumption that the effects of the pressure gradient and body force terms on the oil-film thickness development (see Eq. (1)) are negligible, as compared to that of the skin-friction term, i.e.:

$\varepsilon_{\mathrm{PC}}=\frac{2}{3} \frac{h\left\|\nabla C_{p}-\rho \mathbf{a} / q_{\infty}\right\|}{\left\|C_{f}\right\|} \ll 1$,

where $\varepsilon_{\mathrm{PC}}$ is the ratio of the Poiseuille flow term to the Couette flow term, $q_{\infty}=0.5 \rho_{\infty} u_{\infty}{ }^{2}$ is the freestream dynamic pressure, $\rho_{\infty}$ is the freestream gas density, $u_{\infty}$ is the freestream velocity, $C_{p}$ is the pressure coefficient, and $C_{f}$ is the skin-friction coefficient. The pressure coefficient and the skin-friction coefficient are defined by the freestream dynamic pressure. As discussed in Lee et al. (2018), this assumption is not mandatory for the application of the GLOFSFE, but the effects of the pressure gradient and of the body force terms on the oil-film thickness development should be evaluated to determine if they can be considered negligible; otherwise, they should be measured. 


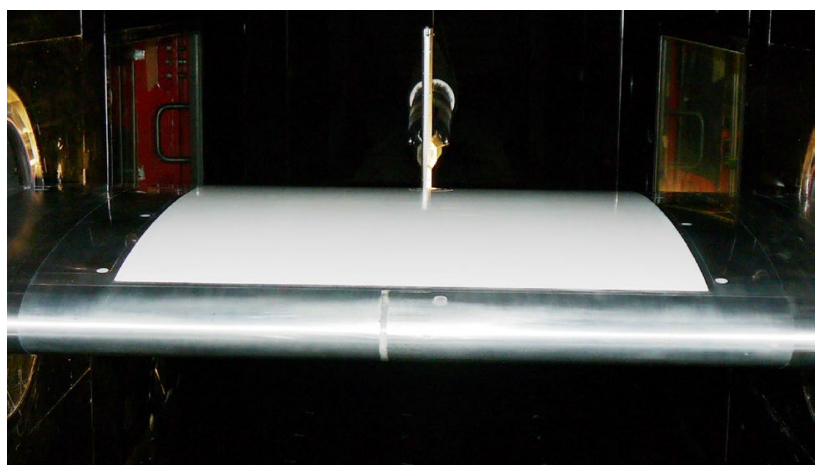

Fig. 1 VA-2 supercritical airfoil model installed in the DNW-TWG test section when looking to the leading edge of the model. The white area on the model surface had been prepared to test different measurement techniques in previous experiments. No base coat is applied in this figure

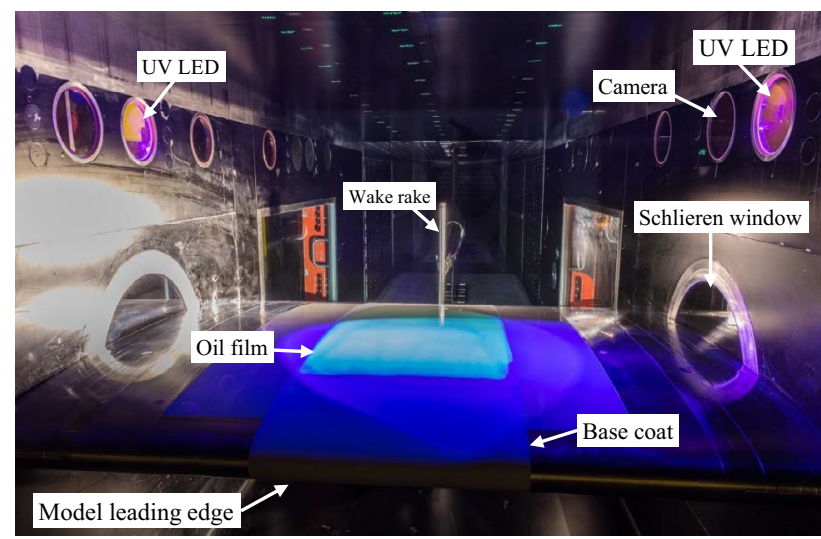

Fig. 2 Airfoil model and measurement techniques installed in the DNW-TWG test section. Ultraviolet light-emitting diodes (UV LEDs) on

\section{Experimental configurations}

\subsection{Wind tunnel, test object, and conventional measurement techniques}

Figures 1 and 2 show the VA-2 supercritical airfoil model of 1-m chord by 1-m span (Krenkel 2012; Leuckert 2012) installed in the DNW-TWG wind tunnel. DNW-TWG is a closed-circuit, variable density wind tunnel (Binder et al. 1992), in which subsonic, transonic, and supersonic flow conditions can be implemented, depending on which one of the three exchangeable test sections is used. In the present study, the experiments were performed in the adaptive-wall test section, which enables subsonic-to-transonic flow conditions to be covered (freestream Mach numbers from $M=0.3$ to 0.9 ). Within this range, the Mach number can be regulated in 0.005 -steps.

The freestream Mach number was determined from the measurements of the flow total pressure and freestream static pressure, according to the isentropic flow equation. The flow total pressure was measured in the DNW-TWG settling chamber, whereas the freestream static pressure was measured using a pressure tap located at the upstream region of the test-section side wall. Both pressures were measured by means of absolute pressure sensors with an accuracy of $\pm 15 \mathrm{~Pa}$. These pressure sensors as well as all other sensors for the measurement of flow parameters were scanned at 330 $\mathrm{Hz}$ for each channel, using a 18-bit data acquisition system. The data were averaged over an integration time of $1 \mathrm{~s}$. During the present investigations, the Mach number was kept constant (within $\Delta M= \pm 0.003$ ) around the set value of 0.72 .

The freestream Reynolds number was determined from the freestream Mach number, the flow total pressure, the flow total temperature, and the freestream dynamic viscosity. The flow total temperature was evaluated as the average of the measurements of four resistance temperature detectors (Pt100) installed in the DNW-TWG settling chamber. The accuracy of the flow total temperature measurement was $\pm 0.2 \mathrm{~K}$. The freestream dynamic viscosity was evaluated via Sutherland's law for the freestream static temperature, which was determined from the freestream Mach number and the flow total temperature according to the isentropic flow equation. In the current work, the uncertainty in the Reynolds number, based on the model chord length $c=1 \mathrm{~m}$, was within $\Delta R e_{c}= \pm 0.0035 \cdot 10^{7}$ ).

As mentioned above, the used DNW-TWG test section has adaptive walls on the upper and lower sides, which allow, in general, interference-free contours to be set. These flexible walls can be deflected via servo motors, with a maximal deflection of the actuators of $120 \mathrm{~mm}$ and an accuracy of $\pm 0.1 \mathrm{~mm}$ (Weiand et al. 2017). The wall adaptation is accomplished by means of a non-iterative Cauchy method based on the pressure distribution measured on the walls and on their deflection (Amecke 1986; Rosemann et al. 1995). The test-section upper and lower walls are equipped with 25 and 23 pressure taps, respectively. The wall pressures were measured by means of electronic pressure scanning modules with an accuracy of $\pm 69 \mathrm{~Pa}$.

In the present work, $x$ is the chord-wise coordinate, positive from the model leading edge to the model trailing edge; $y$ is the span-wise coordinate, positive from the model port side to the model starboard side; and $z$ is the wall-normal coordinate, positive upward. The model rotated around the rotation center at $42.5 \%$ of the chord. The angle of attack AoA of the wind-tunnel model was set by means of a servo drive with an accuracy of $\pm 0.016^{\circ}$.

The airfoil model was instrumented with pressure taps to measure the surface pressure distribution. The pressure 
taps were arranged into three chord-wise rows, located along the model centerline $(y / c=0.5)$ and at the spanwise locations $y / c=0.32$ and $y / c=0.68$. The model surface pressures were measured using electronic pressure scanning modules with an accuracy of $\pm 62 \mathrm{~Pa}$. The windtunnel model was also equipped with two thermocouples to measure the model surface temperature (and thus estimate the oil temperature). The accuracy of the temperature measurement by means of the thermocouples was $\pm 0.3 \mathrm{~K}$. A wake rake was mounted $420 \mathrm{~mm}$ downstream from the airfoil trailing edge for the estimation of the airfoil drag coefficient via wake-deficit integration. In this case, the total pressures measured by the probes installed in the wake rake were measured by means of electronic pressure scanning modules with an accuracy of $\pm 41 \mathrm{~Pa}$.

The examined experimental configuration with a large airfoil model was selected as an optimal condition for the development and validation of measurement techniques, since it enabled investigations at high chord Reynolds numbers with a large measurement surface. However, it was not appropriate for the evaluation of the aerodynamic performance of an airfoil over a broad AoA-range. In particular, the adaptation of the test-section walls could not converge at AoA $>1.3^{\circ}$, because the local flow velocity in the proximity of the upper and lower walls was outside of the application limits of the wall adaptation algorithm (Amecke 1986; Rosemann et al. 1995; Weiand et al. 2017). Moreover, the wake-rake position was too close to the model trailing edge to measure the airfoil drag correctly, since its distance from the model trailing edge should have been at least 0.7 chord (Barlow et al. 1999). Nevertheless, the wake-rake measurements were useful for the estimation of the drag increase due to the oil-film setup, as will be discussed in Sect. 6.2.

A base coat had to be applied to the wind-tunnel model to prevent contamination of the pressure taps due to the oil. Moreover, in preliminary tests, the original (clean) model surface (see Fig. 1) was shown to provide a low signal-tonoise ratio in the GLOF images. A white matte film (Wrap Film Series 1080-M10, from 3M) was applied as the base coat to the airfoil model, thus covering the central row of pressure taps (see Fig. 2). The base coat, which had a thickness of approximately $100 \mu \mathrm{m}$ and a width of $320 \mathrm{~mm}$, was applied to the model from $15 \%$ of the lower side around the leading edge and over the upper side up to the trailing edge. The pressure distribution on the model surface was monitored and measured by the starboard- and port-side pressure taps when the base coat was applied. Otherwise, the central row of pressure taps, which had a higher spatial resolution, was used (see Fig. 4, Sect. 3.4). In addition to the model pressure distribution, a Schlieren photography system (Krenkel 2012) was used to optically identify shock waves in the chord-wise region at approximately $29 \% \leq x / c \leq 56 \%$.

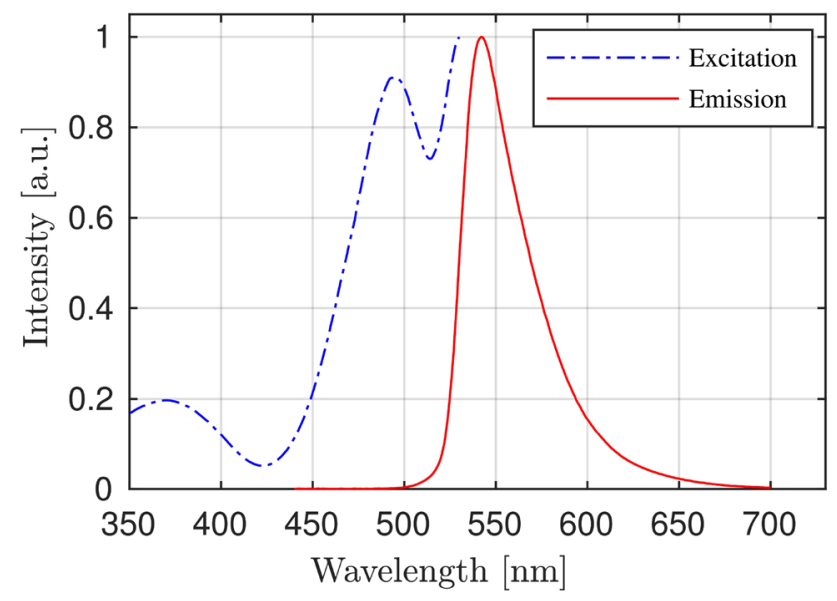

Fig. 3 Excitation and emission spectra of Pyrromethene 567 in methanol solution. Experimental parameters for spectra measurements: $\lambda_{\mathrm{em}}=540 \mathrm{~nm}, \lambda_{\mathrm{ex}}=495 \mathrm{~nm}, \Delta \lambda_{\mathrm{em}}=1 \mathrm{~nm}, \Delta \lambda_{\mathrm{ex}}=20 \mathrm{~nm}$

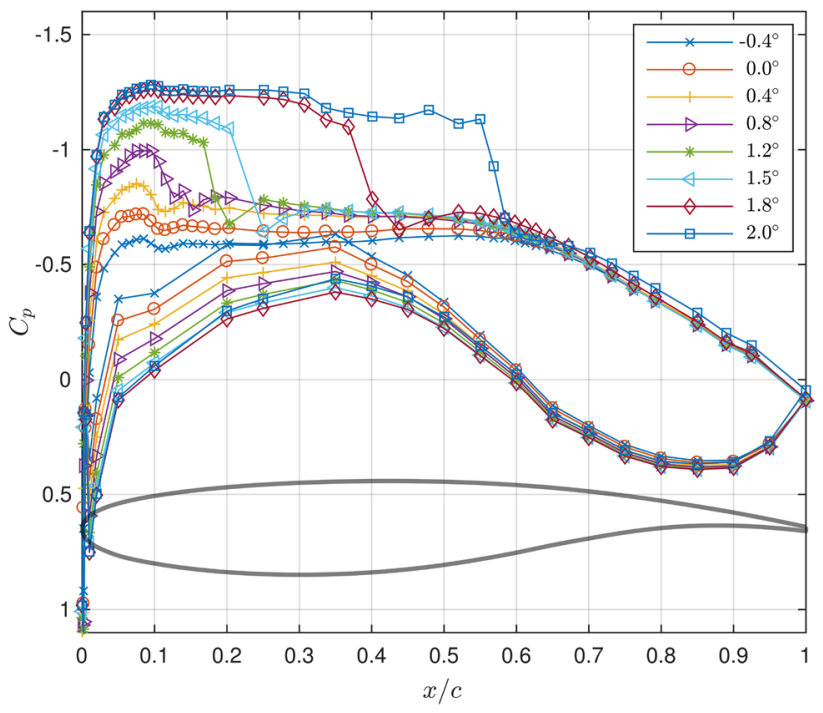

Fig. 4 Distributions of the pressure coefficient $C_{p}$ on the VA-2 supercritical airfoil model at various angles of attack, superimposed with the airfoil profile. Clean configuration (no base coat applied)

An overview of the experimental setup in the DNW-TWG test section will be also provided in Fig. 11 (Sect. 5.3), together with the coordinate system and with an example dataset obtained via both optical and conventional measurement techniques.

\subsection{Luminescent oil}

For the application of the GLOF method (Liu et al. 2008; Husen et al. 2018), a luminescent dye is dissolved into the oil to be applied as a film to the wind-tunnel model surface. When the oil film is illuminated by incident light at 
the dye-excitation wavelength $\lambda_{\mathrm{ex}}$, it emits light at $\lambda_{\mathrm{em}}>\lambda_{\mathrm{ex}}$ (Stokes shift), which can be recorded by a photosensitive device, such as a Charge-Coupled Device (CCD) camera. In the present work, the luminescent oil was made by dissolving a laser dye (Pyrromethene 567, from Exciton) into dimethyl silicone oil (Elbesil Öl B, from L. Böwing) at a weight ratio of approximately $15 \mathrm{ppm}$, which is near saturation. A bottle containing the mixture was placed in an ultrasonic bath with hot water. Ultrasonic waves were applied, thus quickening the dye dissolution. Luminescent oils with kinematic viscosities (at a temperature of $25^{\circ} \mathrm{C}$ ) of $10,000,30,000$, and $60,000 \mathrm{cSt}$ were prepared for the present investigation, because a high oil viscosity was expected to be needed at the considered transonic flow conditions (see Sect. 4.3).

Figure 3 shows the excitation and emission spectra of Pyrromethene 567 in methanol solution, measured by means of a spectrofluorometer. The excitation spectrum was measured with an emission wavelength of $\lambda_{\mathrm{em}}=540 \mathrm{~nm}$, while the emission spectrum was measured with an excitation wavelength of $\lambda_{\mathrm{ex}}=495 \mathrm{~nm}$. The spectral bandwidths of the excitation and emission wavelengths were set to $\Delta \lambda_{\mathrm{ex}}=20 \mathrm{~nm}$ and $\Delta \lambda_{\mathrm{em}}=1 \mathrm{~nm}$, respectively, for both of the spectra. The difference in the spectral bandwidths was due to the difference in the levels of the excitation and emission signals. The larger spectral bandwidth of the excitation wavelength, as compared to that of the emission wavelength, allowed an increase in the signal-to-noise ratio of the measured spectra. An emission peak of $542 \mathrm{~nm}$ and excitation peaks of $370 \mathrm{~nm}$ and $495 \mathrm{~nm}$ can be seen in Fig. 3.

\subsection{Optics}

Optical access at DNW-TWG is available through circular windows on the starboard and port sides of the test section. They have a diameter of $100 \mathrm{~mm}$ and a vertical distance of $420 \mathrm{~mm}$ between the window center and the plane corresponding to the model rotation center. The Schlieren windows are semicircular and have a radius of $162.6 \mathrm{~mm}$, where the center points match the model rotation center.

A UV LED light source (IL-107UV, from HARDsoft), with a peak wavelength of $390 \mathrm{~nm}$, was installed on each side of the test section. These illumination devices were selected to excite the luminescent dye around its first peak ( $\lambda_{\text {ex }}=370 \mathrm{~nm}$, see Sect. 3.2). Although the magnitude of this peak was lower than that of the second peak at $495 \mathrm{~nm}$ (see Fig. 3), the high-power LEDs (which current was set to $15 \mathrm{~A}$ ) enabled the attainment of sufficient intensity of light emitted by the luminescent oil film, while at the same time maintaining a marked wavelength separation between excitation and emission signals. A 14-bit CCD camera (pco.4000, from PCO) was installed to acquire sequential GLOF images. The camera has a resolution of $4008 \times 2672$ pixels, a pixel-sensor size of $9 \mu \mathrm{m}$, and a maximal frame rate with full image size of five frames per second (fps). A manual focus lens with a focal length of $50 \mathrm{~mm}$ (Nikkor $50 \mathrm{~mm} \mathrm{~F} / 1.2 \mathrm{Ai}-\mathrm{S}$, from Nikon) was installed in front of the camera via a Scheimpflug adapter (Scheimpflug mount, from LaVision). The optical setup was adjusted to focus on the model surface area ranging from 9 to $46 \%$ of the chord and from 0.375 to 0.625 of the span. This was the surface region of major interest in the present work: as will be shown in Sect. 6, the main fluid dynamics events on the airfoil upper side (in particular laminar-turbulent transition and shock waves) occurred in this area, which will be named "GLOFSFE-evaluation region" throughout this work.

The separation of the excitation and emission lights was accomplished also by the installation of appropriate optical filters in front of the LEDs and of the camera. Sharp-edged band-pass filters for the wavelength range of $385 \pm 35 \mathrm{~nm}$ were placed in front of the LEDs to block light at lower and higher wavelengths. A 550-nm long-pass filter was installed in front of the camera lens to block the excitation light, while at the same time allowing the light emitted by the oil film to be captured. In fact, the cut-on wavelength of $550 \mathrm{~nm}$ indicates $50 \%$ of the peak transmittance, and the transmittance curve of the used optical filter is relatively smooth. Thus, the emission intensity was sufficiently high for the GLOF measurement, even though the cut-on wavelength was close to the emission peak.

\subsection{Flow conditions}

The flow parameters were a freestream Mach number of 0.72 , a flow total pressure of $80 \mathrm{kPa}$, a flow total temperature of approximately $310 \mathrm{~K}$, and a freestream Reynolds number based on the chord length $c=1 \mathrm{~m}$ of $R e_{c}=10^{7}$. The angle of attack (AoA) was varied as $-0.4,0.0,0.4,0.8$, $1.2,1.5,1.8$, and $2.0^{\circ}$, thus enabling the study of various boundary-layer stability situations on the model upper side. This was, in general, the surface at lower pressure, i.e., with more negative values of the pressure coefficient $C_{p}$. The pressure distributions measured on the clean configuration surfaces (i.e., without the base coat) are shown in Fig. 4, where curves with different colors correspond to different angles of attack. It should be noted here that the pressure distributions on both model surfaces (upper and lower sides) are presented in this figure, but the focus of the present work was on the model upper surface. Therefore, only the upper side pressure distributions will be considered in the following sections, when the discussion will refer to Fig. 4.

The pressure distributions at $\mathrm{AoA}>1.3^{\circ}$ are not expected to be representative for the VA-2 airfoil at free-flight conditions, since the adaptation procedure of the test-section walls did not converge in these cases (see Sect. 3.1). Therefore, pre-defined wall contours were implemented at AoA $>1.3^{\circ}$. The pressure distributions on the model upper 
surface showed, for a significant portion of the chord length, weakly accelerated flow at $\mathrm{AoA}=-0.4^{\circ}$, and a quasi-zero pressure gradient at $\mathrm{AoA}=0.0^{\circ}$; the pressure minimum was enhanced with increasing AoA up to $0.8^{\circ}$, whereas shock waves occurred at larger AoA, with a downstream shift of the shock location with increasing AoA.

\section{Measurement procedure}

\subsection{Measurement parameters}

The evaluation of the requirements on the conditions for the application of the GLOFSFE is presented in this Subsection by following the structure of Sect. 2.3.

\subsubsection{Estimation of the critical limit of roughness}

To estimate the critical limit of surface roughness in the laminar- and turbulent-flow regions, the expected scales of the skin friction were evaluated assuming a flat surface with a zero pressure gradient. This approximation is feasible, because the examined supercritical airfoil was designed to have, on the upper side, a relatively flat pressure distribution for a significant portion of the airfoil chord length (see Fig. 4). Moreover, the incompressible boundary-layer equations are used for the estimation of the skin-friction scales. Although this is generally incorrect for the study of a transonic, compressible flow (such as that considered in this work), it will be shown in Sect. 6.3 that the estimated value of the laminar skin-friction coefficient represented well the actual skin-friction distribution for a significant chord-wise portion of the investigated laminar-flow region. Also in the flat-plate turbulent boundary-layer approximation, the value of the skin-friction coefficient for incompressible flow was expected to be close to the compressible one at the examined flow conditions (Schlichting 1968), since the flow was locally subsonic in most of the turbulentflow regions. In any case, it should be noted here that the following equations were used only for the estimations of the skin-friction scales, whereas the compressible-flow parameters were used in the GLOF data analysis (see Sect. 5.3).

The skin friction coefficient for the laminar region was estimated as follows (Blasius 1908):

$C_{f, \mathrm{~L}}=\frac{0.664}{\operatorname{Re}_{x}^{0.5}}$,

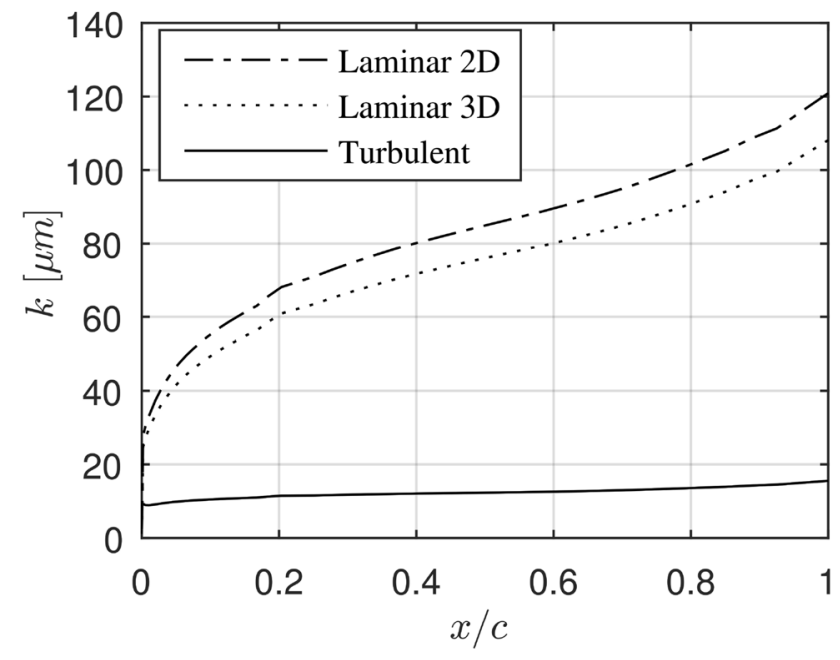

Fig. 5 Chord-wise distributions of the estimated critical limits of surface roughness $k$ on the VA-2 airfoil model at AoA $=1.2^{\circ}$. Estimations based on incompressible flat-plate assumptions and on the surface pressure distribution measured at this AoA

where $R e_{x}$ is the Reynolds number based on the chord-wise distance from the leading edge $x$. On the other hand, the following empirical equation (Schlichting 1968)

$C_{f, \mathrm{~T}}=\left(2 \log _{10} R e_{x}-0.65\right)^{-2.3}$

was employed for the turbulent region. Based on the flow conditions described in Sect. 3.4, the representative skinfriction coefficients $C_{f, \mathrm{~L}}=4 \cdot 10^{-4}$ and $C_{f, \mathrm{~T}}=3 \cdot 10^{-3}$ were estimated, which are the values corresponding to the chordwise location at $x / c=0.30$ on a flat plate for laminar and turbulent flow, respectively. These representative values will be used for the identification of the transition region, which is defined in this study as the region between the locations where the estimated $C_{f}$ became larger than $C_{f, \mathrm{~L}}$ and where $C_{f}$ reached $C_{f, \mathrm{~T}}$.

The critical limits of the surface roughness $k$ in the laminar and turbulent boundary layers were estimated according to Eqs. (11) and (12) with the skin-friction estimations from Eqs. (15) and (16), respectively. Figure 5 shows the chordwise distributions of the estimated critical limits of surface roughness when the pressure data at $\mathrm{AoA}=1.2^{\circ}$ are used. The wall shear stress was estimated as the product of $C_{f}$ from Eqs. (15) or (16) with the local dynamic pressure, which was evaluated from the pressure taps data.

For example, the roughness at $x / c=0.30$ should indicatively satisfy: 
$k \leq \begin{cases}75 \mu \mathrm{m}, & \text { for the laminar region, } 2 \mathrm{D} \text { roughness }\left(R e_{k, \mathrm{cr}}=125\right), \\ 67 \mu \mathrm{m}, & \text { for the laminar region, 3D roughness }\left(R e_{k, \mathrm{cr}}=100\right), \\ 12 \mu \mathrm{m}, & \text { for the turbulent region, }\end{cases}$

where the critical roughness Reynolds numbers $\operatorname{Re}_{k, \mathrm{cr}}=125$ and 100 from Smith and Clutter (1959) and Braslow (1966) were taken as reference critical values for two-dimensional and three-dimensional roughness, respectively.

The larger estimated limit of critical roughness for the laminar-flow region, as compared to that for the turbulent-flow region, may appear as counter-intuitive, but it is related to the different nature of the roughness effect on the boundary layer. As discussed in Sect. 2.3.1, in the laminar-flow region, the effect of a roughness size larger than the critical limit would be to induce premature boundary-layer transition. Essentially, the relevant parameter for the evaluation of this effect in the laminar boundary layer can be considered the relative size of the roughness with respect to the boundary-layer displacement thickness. In contrast, the roughness has an influence on the development of the turbulent boundary layer as soon as it protrudes outside of the viscous sublayer. As studied in Lee et al. (2020c), among others, the relevant parameter for the evaluation of this effect in the turbulent boundary layer is the relative size of the roughness with respect to the thickness of the viscous sublayer. At a certain location on an aerodynamic surface, examined at otherwise the same flow conditions, the viscous sublayer thickness for a fully turbulent boundary layer is indicatively smaller than the displacement thickness of a still laminar boundary layer. This expectation supports the difference in the estimated limits of critical roughness for the laminar- and turbulent-flow regions presented in Eq. (17).

\subsubsection{Determination of the oil dynamic viscosity and estimation of the CFL number}

The dynamic viscosity was determined according to the following equations (Lee et al. 2020c):

$\log _{10} v=763.1 \mathrm{~K} \cdot\left(1 / T-1 / T_{0}\right)+\log _{10} v_{0}$,

and

$\rho=965 \mathrm{~kg} \mathrm{~m}^{-3}-0.860 \mathrm{~kg} \mathrm{~m}^{-3} \mathrm{~K}^{-1} \cdot\left(T-T_{0}\right)$,

where $T_{0}=298.15 \mathrm{~K}$ is the standard oil temperature, at which the kinematic viscosity of the used oil (see Sect. 4.3) is $60,000 \mathrm{cSt}$. For a typical oil temperature of $300 \mathrm{~K}$ in the present investigations, the dynamic viscosity was approximately $56 \mathrm{~Pa} \cdot \mathrm{s}$.

In the wind-tunnel experiments, the camera parameters were set as follows: exposure time of $50 \mathrm{~ms}$, frame rate of
$4.3 \mathrm{fps}$, image size of $3020 \times 1000$ pixels, and spatial resolution of approximately 5600 pixels per meter. With a reference oil-film thickness of $30 \mu \mathrm{m}$, the CFL number expected in the turbulent region was:

$C F L=\frac{\tau h}{\mu} \frac{t_{*}}{x_{*}}=\frac{63 \mathrm{~Pa} 30 \mu \mathrm{m} 5600 \mathrm{~m}^{-1}}{56 \mathrm{~Pa} \cdot \mathrm{s} 4.3 \mathrm{~s}^{-1}}=0.044$,

which would be very small. (In the laminar region, the expected CFL number was even smaller than that in the turbulent region.) The CFL number was therefore expected to be significantly lower than the maximally allowable CFL number introduced in Sect. 2.3.2. However, in some cases, the skin-friction estimations from the present GLOF data in the turbulent regions were significantly larger than these expectations, leading to locally larger estimations of the $\mathrm{CFL}$ number. This aspect will be discussed in the end of Sect. 6.1.

\subsubsection{Evaluation of the pressure gradient and body force effects on the oil-film thickness development}

The ratio $\varepsilon_{\mathrm{PC}}$ [see Eq. (14)] was estimated based on the measured surface pressure distributions and the model geometry. The estimated chord-wise distributions of the numerator of $\varepsilon_{\mathrm{PC}}$ (i.e., the part of this parameter representing the pressure gradient and body force effects) are shown in Fig. 6 for the examined angles of attack. The reference oil-film thickness was assumed to be $100 \mu \mathrm{m}$ as a worst-case

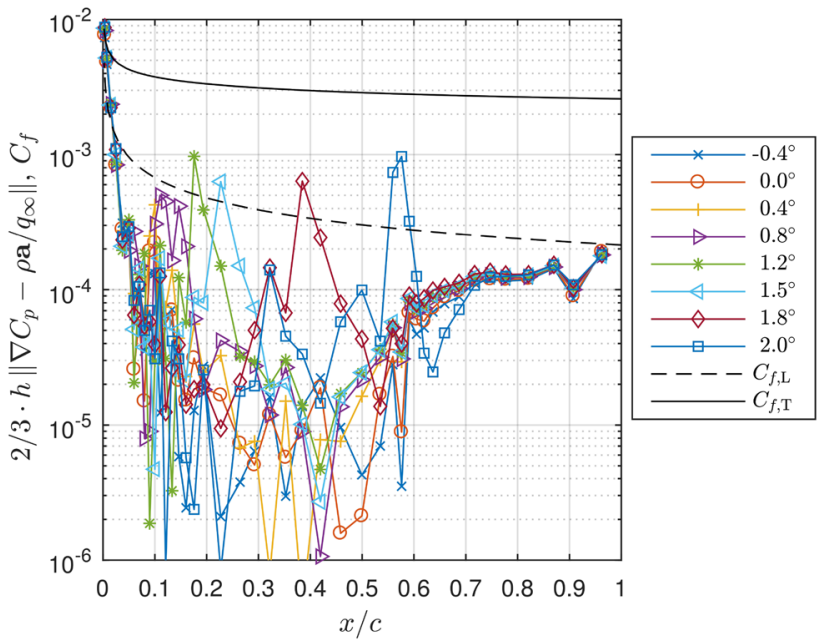

Fig. 6 Pressure gradient and body force contributions with respect to the estimated skin friction (see Eq. (14)) for the examined angles of attack 


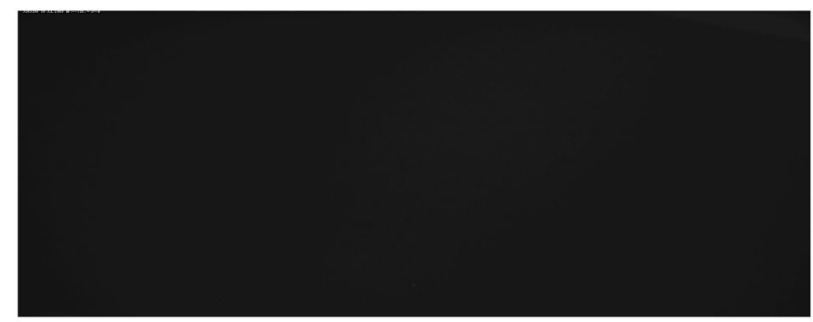

(a) Dark current

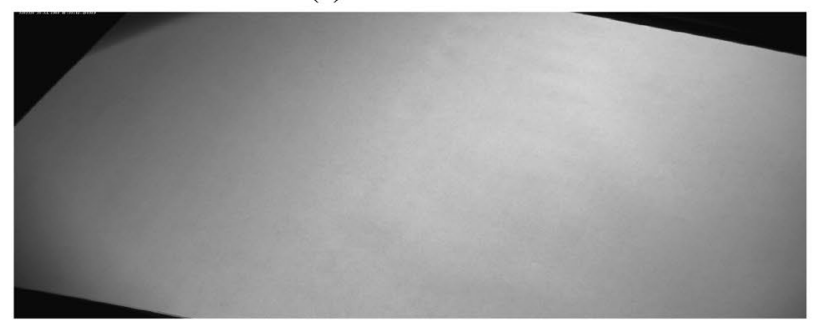

(c) Excitation light distribution

Fig. 7 Representative set of calibration images on the VA-2 airfoil model

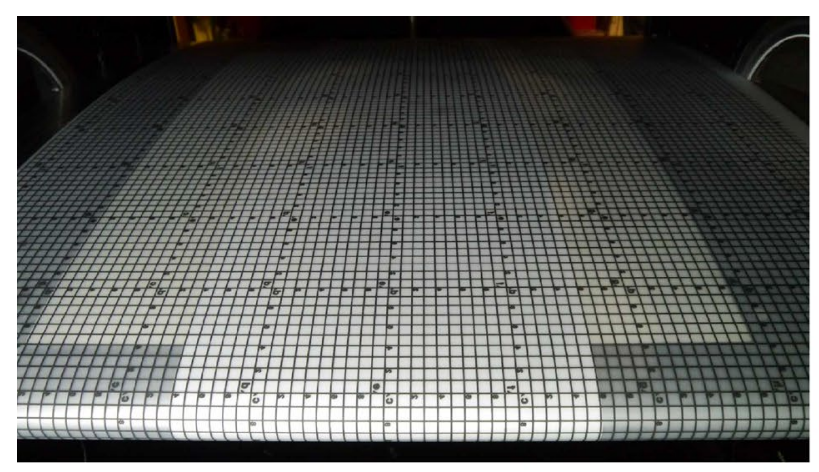

(a) Grid pattern sheet

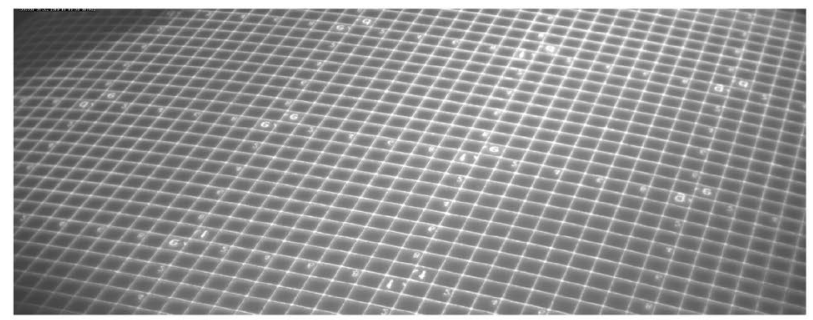

(b) Grid

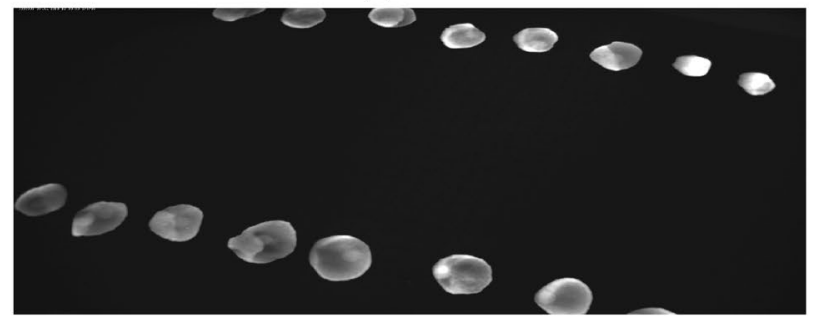

(d) Oil droplets

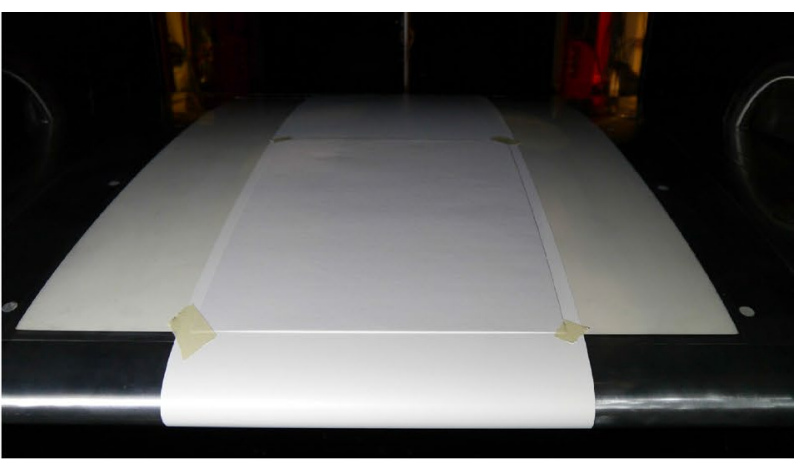

(b) White paper on top of the base coat

Fig. 8 Photographs of the calibration procedure. The leading edge is shown at the bottom of the photographs

scenario. The distributions of the laminar and turbulent skin-friction coefficients from Eqs. (15) and (16) are also presented in Fig. 6. The results show that the effects of the pressure gradient and of the body force terms on the oilfilm thickness development [see Eq. (1)] were sufficiently small in most of the cases, as compared to the effect of the skin-friction term. The neglection of the pressure gradient and body force effects in the GLOFSFE skin-friction estimation was therefore justified, except for the area of very marked pressure gradient related to the shock wave at AoA $\geq 1.2^{\circ}$. The actual pressure gradient was expected to be even more pronounced (as given by a pressure jump of the same strength but on a shorter stream-wise distance) than that observed in the measured pressure distributions, where the local spatial resolution of the available pressure taps was relatively low. Therefore, the pressure gradient and body force effects on the oil-film thickness development were expected to be negligible for most of the cases, except for the narrow region around the shock location at AoA $\geq 1.2^{\circ}$. This aspect will be further discussed in Sect. 6.3.

\subsection{Calibration procedure}

A calibration process is necessary for establishing the relationship between the variables measurable via GLOF and the desired variables, i.e., to move from spatio-temporal image data to the oil-film dynamics. The calibration parameters were determined according to a calibration procedure, which was discussed in detail in Lee et al. $(2018,2020$ c). A set of calibration images (shown in Fig. 7) were acquired with the wind tunnel at rest, as summarized below.

- Dark current images [ $I_{\text {dark }}$ in Eq. (9)] were acquired to account for the influence of residual light and camera 


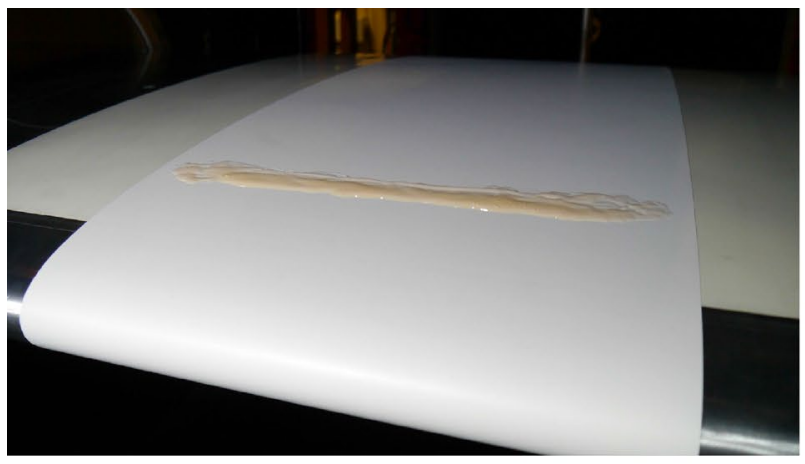

(a) Initial oil-film application

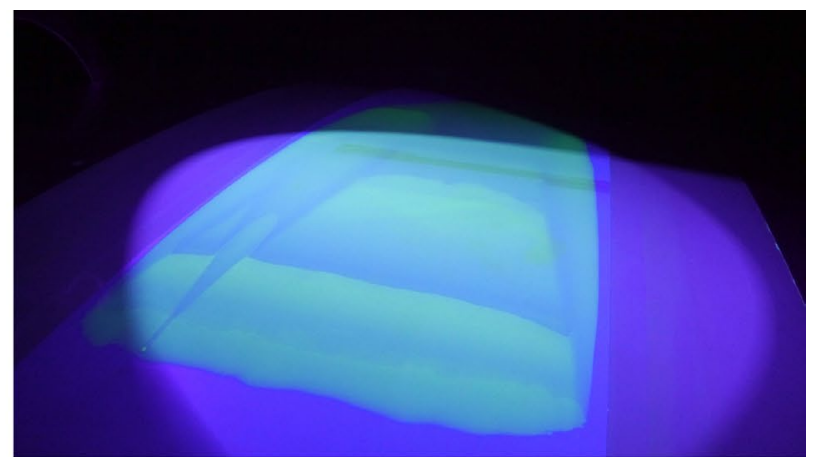

(b) Final oil-film distribution

Fig. 9 Photographs of oil film applied to the model before and after series S2. The leading edge is shown at the bottom of the photographs

electronic noise. These images were taken without any lighting (see Fig. 7a) for each AoA. The exposure time of the camera was the same as that set in the measurement $(50 \mathrm{~ms})$. The averages of the dark current images were then subtracted from the luminescent oil images (see Sect. 5.2).

- To determine the calibration parameters for the projection of the GLOF images into the physical space (see Sects. 5.1 and 5.2), the airfoil model was covered by a flexible plastic sheet with a printed grid pattern, as shown in Fig. 8a. The grid images (Fig. 7b) were taken at each AoA with an adjusted camera exposure time (different from the aforementioned exposure time of the dark current and luminescent oil images). The relative position of the grid with respect to a model reference point (the port side of the trailing edge) was also recorded.

- Excitation light distribution images ( $I_{\text {exc }}$ in Eq. (9)) were acquired by attaching a white paper sheet fully covering the GLOFSFE-evaluation region (see Fig. 8b). In fact, the used white paper included a luminescent dye, and thus allowed the simulation of a homogeneous distribution of luminescent oil film. The $I_{\mathrm{exc}}$ images were taken at each AoA under the excitation light source (see Fig. 7c). As compared to the dark current and luminescent oil images, the exposure time was shortened to $40 \mathrm{~ms}$ to avoid image saturation, since the intensity of the light emitted by the white paper was higher than that of the oil film.

- Oil-droplet images were taken for the determination of the unit thickness $h_{*}$, according to the ratioed image film thickness method (Husen et al. 2018)—see Eq. (7). Oil droplets were dropped onto the surface by means of a micro-pipette (Biomaster 4830, from Eppendorf) with a volume of $20 \mu \mathrm{L}$, and then spread out using compressed air. The oil-droplet images (Fig. 7d) at each AoA were acquired with the same exposure time as that of the dark current and luminescent oil images.

\subsection{Wind-tunnel operation sequence}

The GLOF measurements were conducted as follows. Before wind-tunnel operation, the luminescent oil was applied to the upstream area of the GLOFSFE-evaluation region, as shown in Fig. 9a. The oil was not applied further upstream of the GLOFSFE-evaluation region, since the leading-edge area would have been particularly sensitive to surface roughness (see Sect. 4.1.1). An oil with a high kinematic viscosity of 60,000 cSt (at a temperature of $25^{\circ} \mathrm{C}$ ) was selected. This choice was dictated by the necessity to avoid that the oil would drop during the wind-tunnel preparation time (for example, during the safety check and the wind-tunnel pressure adjustment) and that the oil would separate from the model trailing edge during the runs, thus damaging the wake-rake probes or contaminating the wind tunnel. After the wind-tunnel operation was started, the oil film spread during the flow acceleration. The oil-film thickness was visually monitored during the spreading process. The image acquisition was initiated when the oil covered the GLOFSFE-evaluation region and, at the same time, the image intensity was below saturation. After acquisition of a set of 241 sequential images, the angle of attack was varied, and the image recording was repeated as soon as stable flow conditions were attained again. The series of measurements was then terminated before the oil film reached the model trailing edge, thus avoiding damages of the wake-rake probes or contamination of the wind tunnel. A final oil-film distribution is shown in Fig. 9b.

Each wind-tunnel operation was called a series. Four series with various orders of AoA were conducted (S1 to S4), as described below and summarized in Table 1. The different series were run to investigate the most appropriate starting and running conditions for GLOF measurements in DNW-TWG, and enabled an evaluation of the repeatability of the GLOFSFE estimations, as discussed in Sect. 6.1. 
Table 1 Wind-tunnel operation sequence

\begin{tabular}{lllll}
\hline Series & AoA $\left[^{\circ}\right]$ & & $\begin{array}{l}\text { Initial oil position } \\
{[\%]}\end{array}$ & New base coat \\
\hline S1 & -0.4 to 2.0, increase & 1.8 to -0.4, decrease & 9 & Yes \\
S2 & 1.2 to 2.0, increase & & 9 & Yes \\
S3 & 2.0 to 1.2, decrease & -0.4 to 0.8, increase & 20 & No \\
S4 & 1.2 to 2.0, increase & -0.4 to 0.0, increase & $20-40$ & No \\
\hline
\end{tabular}

- The oil was placed approximately at the upstream limit of the GLOFSFE-evaluation region, i.e., at $x / c \sim 9 \%$, prior to series $\mathrm{S} 1$; in this series, the AoA was increased from $-0.4^{\circ}$ to $2.0^{\circ}$ (S1-01 to S1-08), and then decreased back to $-0.4^{\circ}$ (S1-09 to $\left.\mathrm{S} 1-15\right)$.

- After the base coat had been exchanged, before series S2, the oil was applied at approximately the same location as that of series $\mathrm{S} 1$. The AoA was varied from $1.2^{\circ}$ to $2.0^{\circ}$ (S2-01 to S2-04) in series $\mathrm{S} 2$.

- After cleaning the oil, the oil application position was then moved to approximately $20 \%$ of the chord, and, in series S3, the AoA was varied from $2.0^{\circ}$ to $1.2^{\circ}$ (S3-01 to $\mathrm{S} 3-04$ ), and then from $-0.4^{\circ}$ to $0.8^{\circ}$ (S3-05 to S3-08). The base coat was not further exchanged after series $\mathrm{S} 2$.

- After series S3, the oil was directly added to the investigated surface and, before the wind tunnel was operated again, spread out by means of compressed air. In the final series $\mathrm{S} 4$, the AoA was varied from $1.2^{\circ}$ to $2.0^{\circ}$ (S4-01 to $\mathrm{S} 4-04$ ), and then from $-0.4^{\circ}$ to $0.0^{\circ}$ (S4-05 to $\mathrm{S} 4-06$ ).

\section{Data processing}

\subsection{Camera calibration}

The camera parameters, i.e., the intrinsic parameters (focal length, scale factor, principal point, and radial and tangential distortions) and the extrinsic parameters (camera location and orientation angles), were obtained via camera calibration. The camera calibration parameters were determined by pairs of three-dimensional space coordinates of the model surface and two-dimensional image coordinates of the grid images (see Sect. 4.2). These coordinate pairs were provided by the grid images at the various AoAs, where the image coordinates were obtained by reading the grid image points. The corresponding model surface coordinates were converted to the spatial coordinates, which were determined by the model geometry, the model installation position, and the AoA. The camera calibration was carried out using a MATLAB code by Heikkila (2000).

\subsection{Image projection}

After subtraction of the averaged dark current images, all images were projected onto the three-dimensional model surface. The target model surface coordinates were set to the GLOFSFE-evaluation region, i.e., the area ranging from $x / c=9 \%$ to $46 \%$ and from $y / c=0.375$ to 0.625 (see Sect. 3.3); the resulting pixel density was 5600 pixels per meter. The corresponding image coordinates were obtained from Eq. (8), and the image value was calculated by bicubic interpolation. The projected calibration images and a representative oil-film image are shown in Fig. 10.

\subsection{GLOF image analysis and flow-data integration}

The time-sequential oil-film thickness distributions in the model surface domain were analyzed using the GLOFSFE code (Lee and Liu 2018), written in MATLAB. All 241 luminescent oil images acquired for each data point were paired into 240 image pairs, and then, an average skinfriction field was obtained for each data point, as described in Sect. 2.1. The oil-film thickness $h$ was evaluated according to Eq. (9), where $h_{*}$ was obtained from the oil-droplet images (see Sect. 4.2) via Eq. (7), and the distribution of the angle $\theta_{P}$ was calculated from Eq. (10); here, the normal vector $\mathbf{n}$ was obtained from the model geometry data, and $\mathbf{v}_{\text {camera }}$ was calculated from the optical center point of the camera. The range of $\theta_{P}$ was from $57^{\circ}$ to $68^{\circ}$; therefore, the oil-thickness resolution was approximately double, as compared to that for $\theta_{P}=0^{\circ}$ (Husen et al. 2018).

The estimated skin friction $\hat{\tau}$ was calculated from Eq. (3), where $\hat{\tilde{\tau}}$ was obtained from the GLOF images [see Eqs. (4)-(6)] using the aforementioned GLOFSFEevaluation code. The areas where the given data were illposed were eliminated from the evaluation process. The ill-posed nodes were identified at the locations where the determinant of the system (Lee et al. 2020c) was lower than a threshold, which was four times the double-precision floating-point relative accuracy. It should be emphasized here that ill-posed nodes were therefore detected also when the estimated wall shear stress approached zero, i.e., 


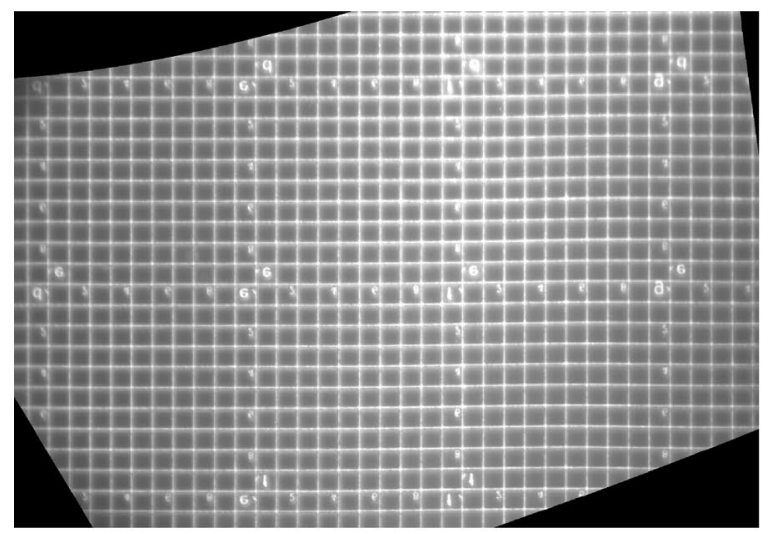

(a) Grid

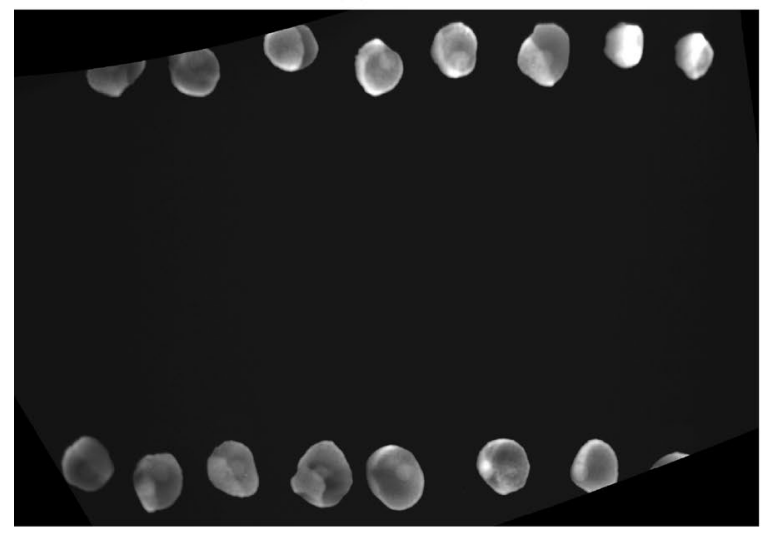

(c) Oil droplets

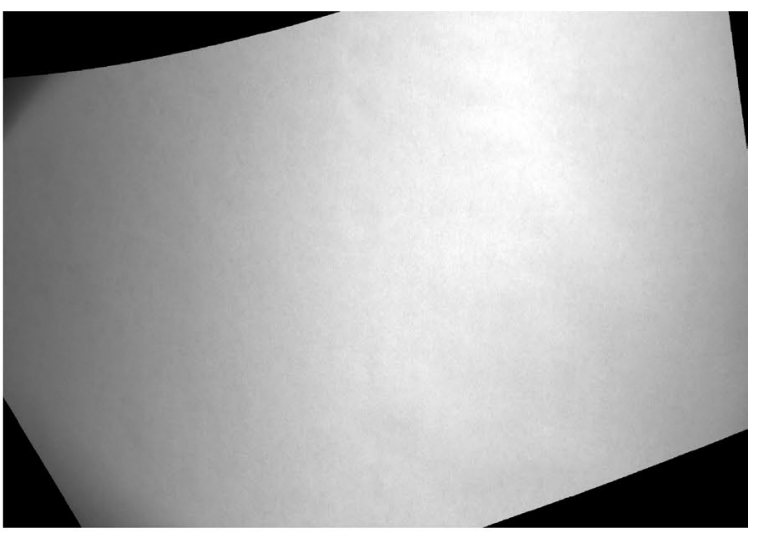

(b) Excitation distribution

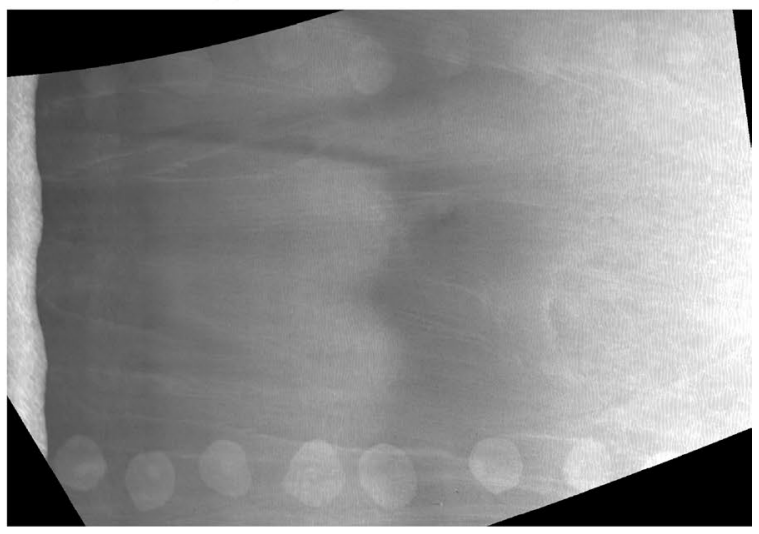

(d) Oil distribution

Fig. 10 Representative set of projected images on the model surface at $M=0.72, R e_{c}=10^{7}$, and $\mathrm{A} o A=0.8^{\circ}(\mathrm{S} 1-04)$

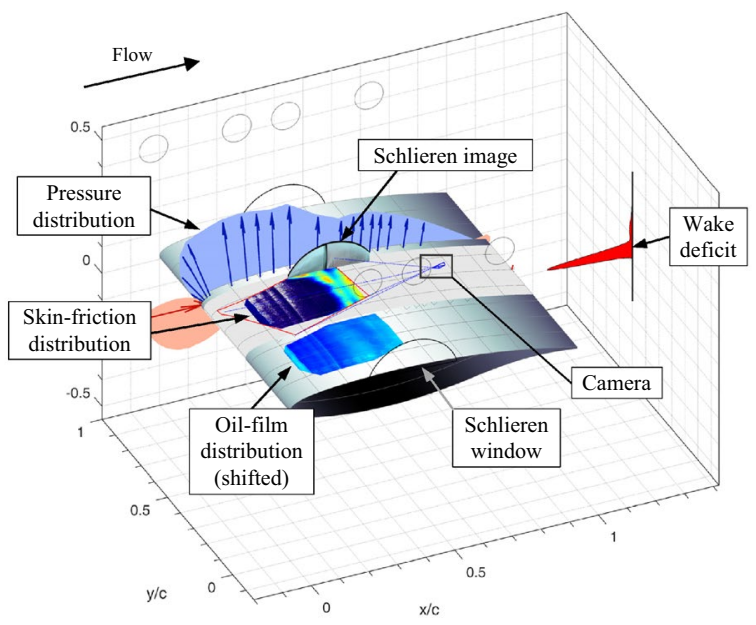

Fig. 11 Integrated dataset of GLOFSFE and flow measurement results for the test section and the model coordinates at $M=0.72$, $R e_{c}=10^{7}$, and $\mathrm{AoA}=1.8^{\circ}(\mathrm{S} 1-07)$ also at separation and reattachment locations as well as in their vicinity. This aspect will be discussed in Sect. 6.

Figure 11 shows the GLOFSFE results (estimated oilfilm and skin-friction distributions) for a representative data point at $M=0.72, R e_{c}=10^{7}$, and AoA $=1.8^{\circ}(\mathrm{S} 1-07$, see Sect. 6). In this figure, the pressure distribution on the model surface, the wake deficit, the camera position from the camera calibration, the locations of the Schlieren windows, and the Schlieren image are also shown.

The oil-film thickness in wall units $h^{+}$was evaluated from the estimated oil-film thickness $h$ and skin-friction $\hat{\tau}$ (Lee et al. 2020c):

$\widehat{h^{+}}=h \frac{\left(\hat{\tau} / \rho_{\text {air }}\right)^{0.5}}{v_{\text {air }}}$,

where $\rho_{\text {air }}$ is the air density, and $v_{\text {air }}$ is the air kinematic viscosity. The air properties $\rho_{\text {air }}$ and $v_{\text {air }}$ were computed from the measured surface pressure distribution and flow total quantities, according to the isentropic flow equations. 


\subsection{Estimation of the uncertainties}

A thorough evaluation of the GLOF measurement uncertainties was presented in Lee et al. (2020c). Grounding on that work and on the skin-friction estimation presented in Eqs. (3) and (7), the following uncertainty sources are reviewed for the present study: oil dynamic viscosity, unit length, unit time, calibration oil-droplet volume, calibration image, and normalized skin friction.

- The uncertainty in the oil dynamic viscosity $\Delta \mu$ was estimated from the model surface temperature variation. The adiabatic-wall temperature variation in the GLOFSFEevaluation region with laminar flow (the area where quantitative skin-friction estimations could be carried out, see Sect. 6.1), evaluated on the basis of the measured surface pressure distribution, flow total temperature, and freestream Mach number, was within $\pm 1.1 \mathrm{~K}$ for all examined test conditions. The surface temperature variation in the considered evaluation region was expected to be even less than the adiabatic-wall temperature variation; for a conservative estimation, the uncertainty in the oil temperature was assumed to be $\Delta T= \pm 1.1 \mathrm{~K}$. Incidentally, this is the same uncertainty in the oil temperature as that reported in Lee et al. (2020c), where it corresponded to an uncertainty in the oil dynamic viscosity of $\pm 2.3 \%$. This can be taken as a conservative estimation of the uncertainty in the oil dynamic viscosity in the present work.

- The uncertainty in the unit length $\Delta x_{*}$ depends on the camera pixel density, on the geometry of the surface, on the camera angle $\theta_{P}$, on the camera lens distortion, and on the camera calibration and image projection process (see Sects. 5.1 and 5.2). The resulting estimation of the uncertainty in the unit length was within $\pm 0.5 \%$.

- The uncertainty in the unit time $\Delta t_{*}$ is determined by the camera timing accuracy. In the present work, $\Delta t_{*}$ was estimated to be even smaller than the values reported in Lee et al. (2020c) (within $\pm 0.02 \%$ ) and was therefore regarded as negligible.

- The uncertainty in the unit oil-film thickness $h_{*}$ depends on $\Delta x_{*}$ (discussed above), on the uncertainty in the calibration (ratioed) image $\Delta \overline{r_{\text {cal }}}$ and on the uncertainty in the oil-droplet volume $\Delta v_{\text {droplet. }}$. Since the ratioed image film thickness method was applied in the same manner as in Lee et al. (2020c), the uncertainty in the oil-droplet volume was estimated as $\Delta v_{\text {droplet }}= \pm 1.65 \%$, whereas the uncertainty in the calibration image could be considered as negligible $\left(\Delta \overline{r_{\text {cal }}}\right.$ within $\left.\pm 0.003 \%\right)$.

- As discussed in Lee et al. (2018, 2020c), the uncertainty in the normalized skin friction $\Delta \hat{\tilde{\tau}}$ is determined by the GLOF image noise, the number of GLOF image pairs, and the "image characteristics". This latter definition comprehends several factors, such as the offset error related to the base coat influence on the GLOF measurements (see Sect. 6.1) and the investigated flow conditions. In the present study, it was not possible to evaluate systematically $\Delta \hat{\tilde{\tau}}$ because of the extremely challenging transonic wind-tunnel environment. Challenges for the application of the GLOF measurement technique in the continuously driven DNW-TWG wind tunnel were, among others, the accessibility for the oil application, the time needed to prepare the facility for wind-tunnel operation, and the time necessary to attain stable flow conditions (see Sect. 4.3). The issues that limited the repeatability of the GLOF measurements are described and discussed in Sect. 6.1.

An estimation for $\Delta \hat{\tilde{\tau}}$ in this work may be $\pm 22.6 \%$, based on the worst-case scenario presented in Lee et al. (2018).

In any case, an estimation of the overall uncertainty $\Delta C_{f x}$ in the skin-friction coefficient determined via GLOFSFE will be provided in Sect. 6.1.

\section{Results and discussion}

\subsection{Repeatability of the results and evaluation of the GLOFSFE estimations}

The estimations obtained from the GLOF image analysis of all data points examined in the present work are summarized in Figs. 12, 13, 14 and 15. In general, the variables will be presented without the accent ${ }^{\wedge}$, since it will be specified that the related quantities are estimated via GLOFSFE. The estimated, average oil-film thickness distributions $\bar{h}$ and the coefficients of determination $R^{2}$ (Lee et al. 2018, 2020c) are shown in Figs. 12 and 13, respectively. The chord-wise skin-friction distributions $C_{f x}$ estimated via GLOFSFE are presented in Fig. 14. In these three figures, the gray-masked areas indicate the regions where the GLOF data were unavailable or where the nodes were identified as ill-posed (see Sect. 5.3). Figure 15 shows $C_{f x}$ profiles, obtained from Fig. 14 as averages along the span-wise regions where the $C_{f x}$ and $\bar{h}$ distributions were essentially two-dimensional.

One of the major challenges of GLOF measurements at the examined test conditions becomes apparent when Figs. 12 and 13 are analyzed. The areas with high $R^{2}$ values (i.e., where the data are well represented by the LLS regression) correspond to the regions where the oil film was thick. In these areas, however, the flow was turbulent, and the oil-film thickness was larger than the thickness of the viscous sublayer (see Sects. 2.3.1 and 4.1.1), leading to an increase in the skin friction in Fig. 14, as compared to the clean surface. The increased skin friction was therefore due to a physical effect (the interaction between the oil film and 


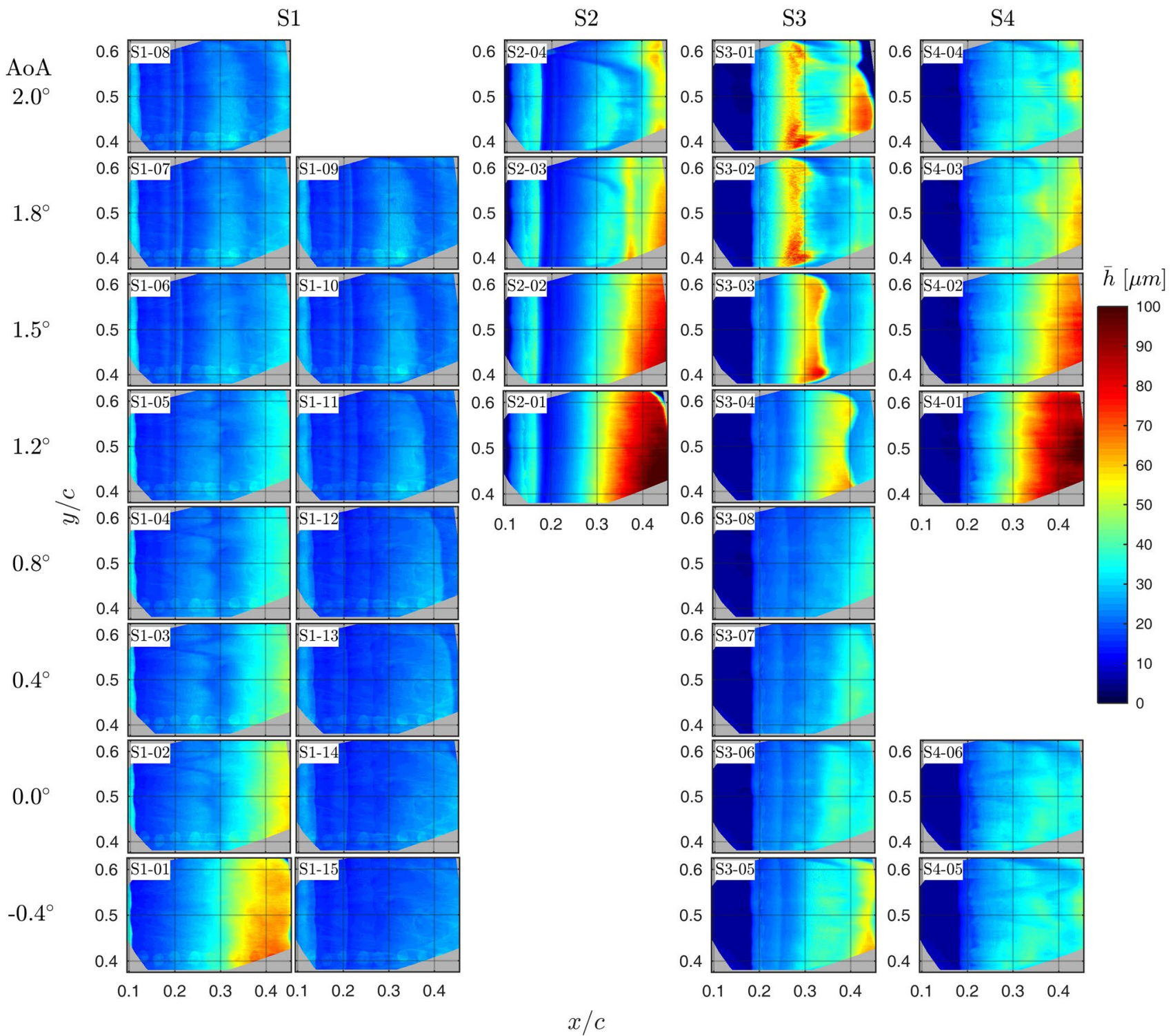

Fig. 12 Estimated oil-film thickness distribution on the GLOFSFE-evaluation region for all examined data points. The gray areas indicate masked areas due to missing image data

the boundary layer), and not to errors in the GLOF image analysis or in the numerical processing. As an extreme case, the maximal value of the estimated $C_{f}$ in the turbulentflow regions was larger than the reference value $C_{f, \mathrm{~T}}$ (see Sect. 4.1.1) by a factor of 36. In contrast, areas with low $R^{2}$ values (i.e., where the LLS regression was poor) correspond to the regions where the oil film was thin. As discussed in Lee et al. (2020c), the thinner oil film leads to increased uncertainties due to the image noise and the image processing, and thus to a reduction in the coefficient of determination and in the confidence of the skin-friction estimation. This affected the estimations obtained in the laminar-flow regions, where the oil film was thin. Nevertheless, the oilfilm thickness in these regions was below the critical limit of roughness discussed in Sects. 2.3.1 and 4.1.1, thus allowing a quantitative estimation of the laminar skin-friction coefficient and of the location of transition onset, which will be presented below as well as in Sect. 6.3.

A dedicated discussion is considered necessary for the data points with the expected presence of laminar separation bubbles. In fact, at the larger angles of attack $\left(\mathrm{AoA} \geq 0.8^{\circ}\right)$, the laminar boundary layer was expected to undergo separation as it encountered a marked adverse pressure gradient, particularly pronounced at $\mathrm{AoA} \geq 1.2^{\circ}$ (see Fig. 4). The strong amplification of disturbances within a separated boundary layer was expected to lead to transition in the separated shear layer, with the resulting turbulent motion eventually inducing the reattachment to 
S1

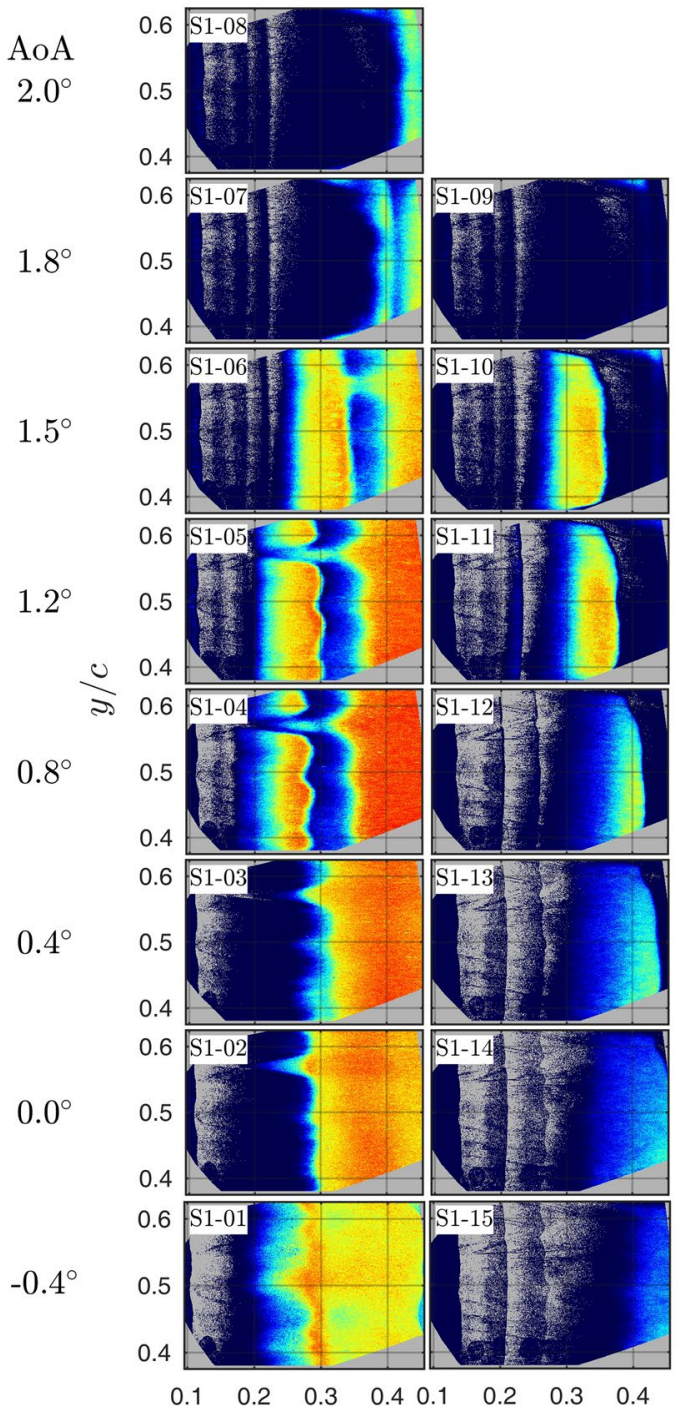

S2

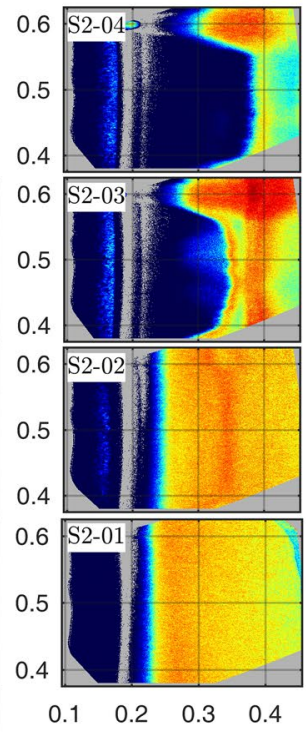

0.6
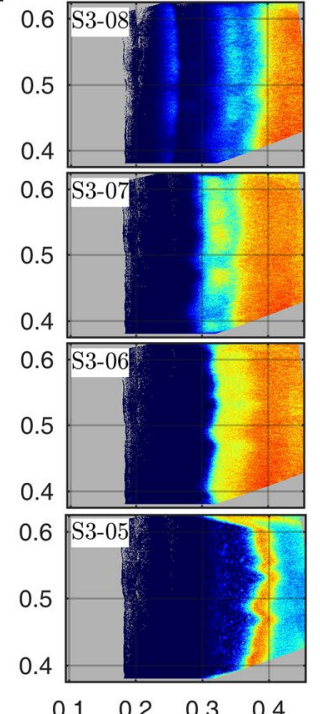

$\begin{array}{llll}0.1 & 0.2 & 0.3 & 0.4\end{array}$
S4

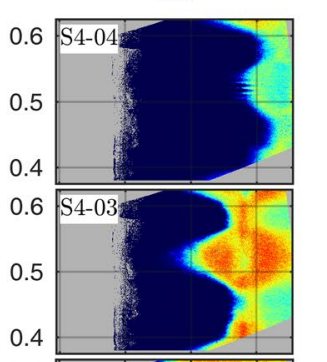

\begin{tabular}{ll}
$0 . 6 \longdiv { 5 4 - 0 2 } \quad R ^ { 2 }$ \\
\hline
\end{tabular}

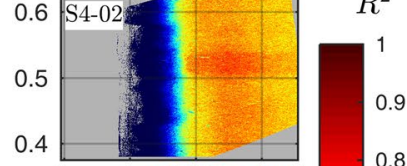

$0 . 6 \longdiv { \mathrm { S } 4 - 0 1 }$

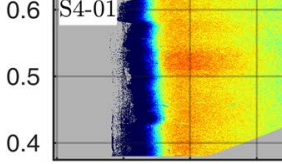

$\begin{array}{llll}0.1 & 0.2 & 0.3 & 0.4\end{array}$
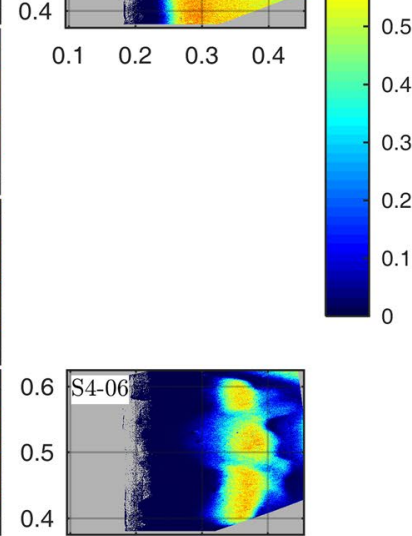

0.6 S4-05

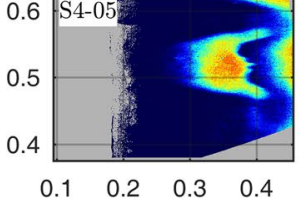

$x / c$

Fig. 13 Coefficient of determination $R^{2}$ of the estimated normalized skin-friction $\tilde{\tau}$ extracted from the GLOF image analysis for all examined data points. Gray areas indicate masked areas due to missing image or ill-posed nodes

the surface and the formation of a laminar separation bubble (see Miozzi et al. (2019), among others). The critical lines (i.e., the separation and reattachment lines), as well as the areas in their vicinity, are regions where the wall shear stress is zero or nearly zero. As mentioned in Sect. 5.3, the nodes in regions of vanishing wall shear stress are detected as ill-posed in the GLOFSFE code. The graymasked stripes, essentially oriented in the span-wise direction, visible in Figs. 13 and 14 at approximately $x / c<30 \%$ (data series S1 and S2), very likely originated from critical lines, and most probably from the reattachment lines. The stream-wise position and extent of the gray-masked stripes was probably determined by both physical factors and time history of the oil-film. This latter influence will be discussed below, focusing on the presence of multiple gray-masked stripes in various data points of series S1 and $\mathrm{S} 2$. With regard to the time-averaged representation of a laminar separation bubble, the reverse-flow region downstream of the separation location is known as a region of low, negative skin friction, which is also named "dead-air" or "dead-water" region (see Costantini et al. (2019) and Miozzi et al. (2019), among others). Also the low value of the negative skin friction in the dead-air region could not be resolved via GLOFSFE at the present experimental conditions, thus possibly contributing to the stream-wise extent of the gray-masked stripes. Between the dead-air region 


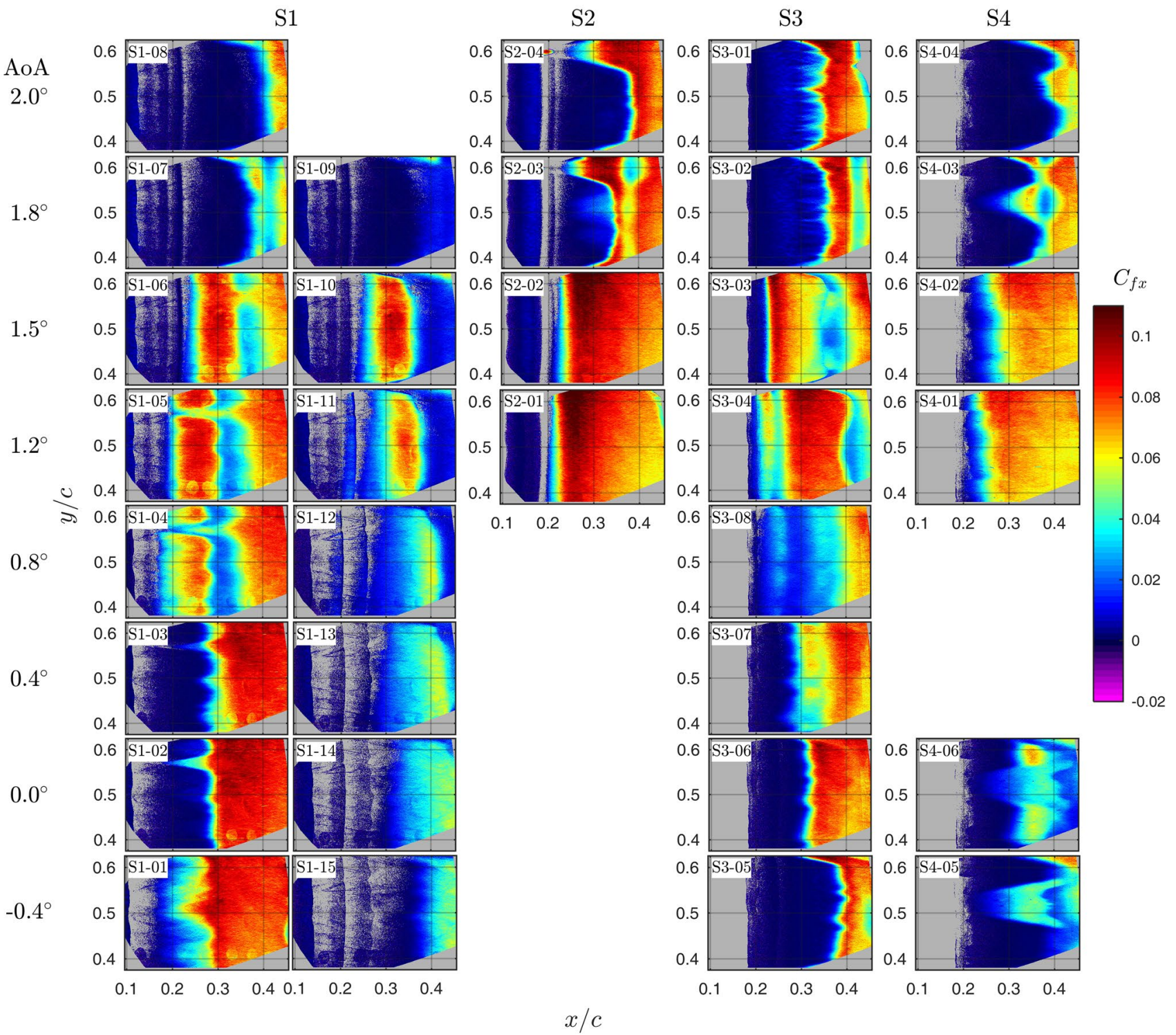

Fig. 14 Estimated chord-wise skin-friction distributions on the airfoil model at $M=0.72$ and $R e_{c}=10^{7}$ for all examined data points. The gray areas indicate the masked areas due to missing image data or ill-posed nodes

and the reattachment location, a region of more intense reverse flow is expected within the laminar separation bubble, with larger negative wall shear stress culminating in a negative skin-friction peak upstream of reattachment. The large skin friction in the separated flow region upstream of reattachment (as well as in the turbulent region downstream of the reattachment location) is expected to be very efficient in removing oil from the reattachment location area. This was very likely the reason for the formation of stripes of very low oil-film thickness, which can be seen in Fig. 12. Their chord-wise locations approximately matched those of the gray-masked stripes in Figs. 13 and 14, thus indicating that the very low GLOF signal was most probably the cause for the unattainability of skin-friction estimations in these regions. Moreover, laminar separation bubbles are also expected to exhibit significant unsteadiness, especially in the region close to the reattachment location. This also probably contributed to the difficulty of detecting the negative skin friction values within the laminar separation bubbles, and especially upstream of the reattachment location.

As can be seen in Figs. 12, 13, 14 and 15, the repeatability of the results was limited. This was due to the following issues:

- Influence of the initial oil position and of the time history of the oil film

When the measurements are started, the skin friction increases along with the flow direction, $\partial \tau / \partial x>0$, and the oil film becomes thinner, 
Fig. 15 Chord-wise skinfriction coefficient profiles $C_{f x}$ at $M=0.72$ and $R e_{c}=10^{7}$ for all examined data points, obtained as averages along the span-wise regions where the $C_{f x}$ and $h$ distributions were essentially two-dimensional. The reference value for the laminar skin-friction coefficient $C_{f, \mathrm{~L}}$ (dash-dotted black line) is also shown
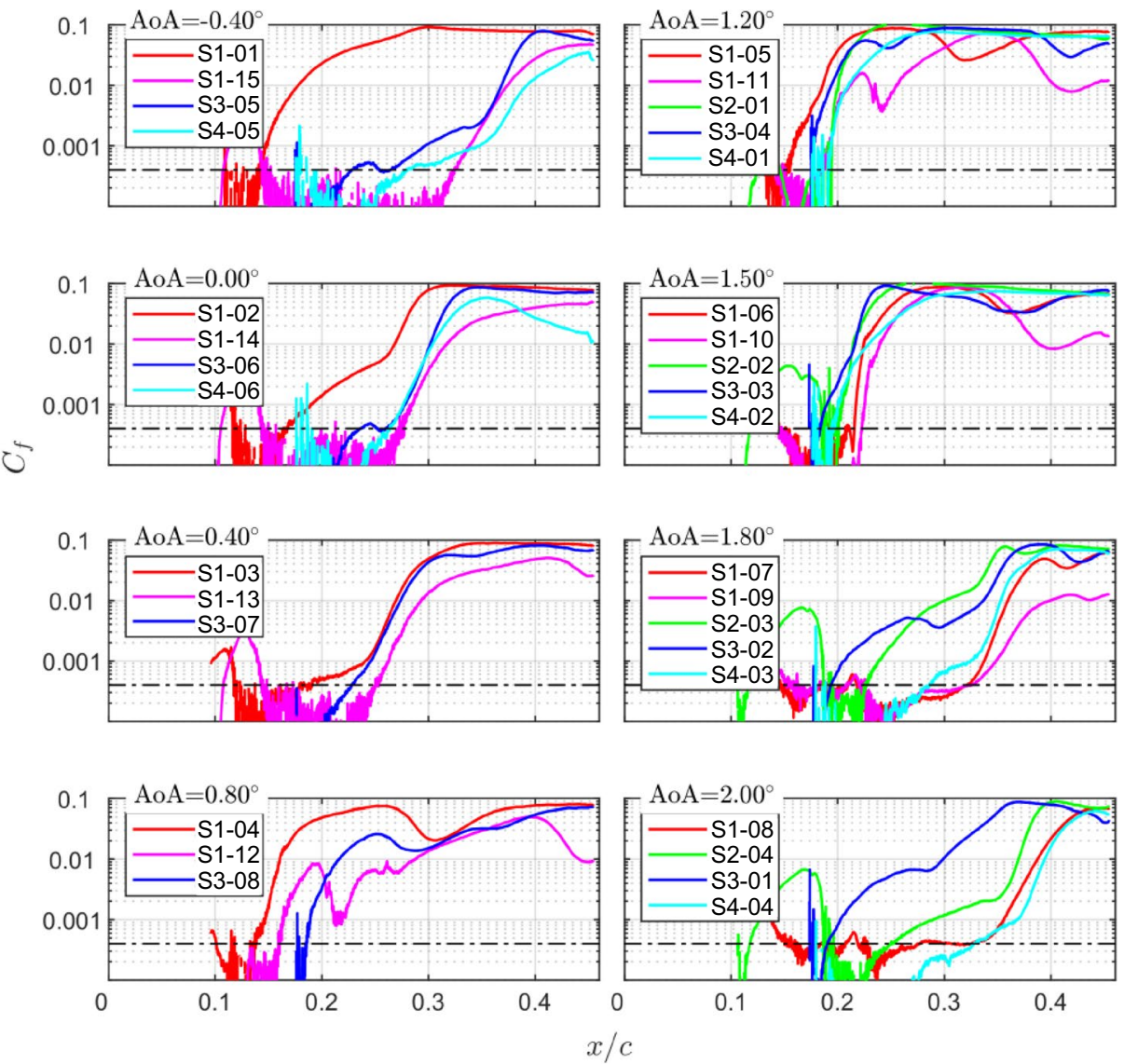

$\partial h / \partial t=-h^{2} / 2 \mu \cdot \partial \tau / \partial x<0$. In series S1 and S2, the oil film positioned at $9 \%$ of the chord was affected by the stream-wise pressure gradient (see the pressure distribution in Fig. 4), which led to the formation of an oil 'bump'. This can be observed in Figs. $9 \mathrm{~b}$ and 12 for the series S2, which was terminated after only four data points. The oil 'bump' formed at $10 \%<x / c<20 \%$ induced a 'bump' also in the estimated skin-friction distributions of series S2, which can be observed in Fig. 14 and, even more clearly, in Fig. 15 (green lines). In addition to this local effect, the oil 'bump' also induced premature transition at $\mathrm{AoA}=1.8^{\circ}$ and $2.0^{\circ}$ (data points S2-03 and S2-04). (As introduced in Sect. 4.1.1, the position of transition onset was identified in the present work at the location where $C_{f}$ became larger than the reference value for laminar flow $C_{f, \mathrm{~L}}=4 \cdot 10^{-4}$.) At AoA $=1.2^{\circ}$ and $1.5^{\circ}$ (data points S2-01 and S2-02), the bump effect on boundary-layer transition was negligible, since the chord-wise distance between the oil 'bump' and the location of natural transition onset was small. In series S1, the oil 'bump' formed at $x / c \sim 10 \%$, and its height was reduced during the conduction of this first series. Nevertheless, a difference in the transition position between the data points at $\mathrm{AoA}=-0.4^{\circ}, 0.0^{\circ}$ and $0.4^{\circ}$ (S1-01 to S1-03 vs. S1-15 to S1-13) can be seen. At these angles of attack, the boundary-layer stability situations were more sensitive to the effect of the bump (see Costantini et al. (2019)), and transition occurred more upstream for the data points S1-01 to S1-03, as compared to the cases S1-15 to S1-13. This effect of the oil 'bump' can be clearly seen in Fig. 15 (red vs. magenta lines). At AoA $\geq 0.8^{\circ}$, the influence of the oil 'bump' on the transition weakened with increasing angle of attack (until it essentially vanished at $\mathrm{AoA} \geq 1.5^{\circ}$ ), because the boundary-layer stability situations became less sensitive to the bump effect. However, a higher oil 'bump' did induce premature transition at $\mathrm{AoA}=1.8^{\circ}$ and $2.0^{\circ}$ in series $\mathrm{S} 3$, where the 'bump' formed at $25 \%<x / c<30 \%$. As can be seen in Figs. 14 and 15, transition occurred at a more upstream location for the data points S3-01 and S3-02, as compared to the data points from series $\mathrm{S} 1$. As the angle of attack was further reduced to $\mathrm{AoA}=1.5^{\circ}$ and $1.2^{\circ}$ (data points S3-03 and S3-04), the oil 'bump' moved into a more downstream location (see Fig. 12), i.e., downstream of the location of natural transition onset. Therefore, the transition location at these two angles of attack in series S3 was essentially the same as that found in series S1 and S2 (see Fig. 15). 
As an example of the influence of the time history of the oil film on the skin-friction estimations in the turbulent-flow regions, it can be seen that a oil 'valley' formed in series S1 at $x / c>25 \%$ starting from data point $\mathrm{S} 1-03$, probably induced by the change in the transition location between the data points S1-02 and S1-03. The oil 'valley' led to a 'valley' also in the skin-friction distributions estimated in the turbulent-flow regions at $0.8^{\circ} \leq \mathrm{AoA} \leq 1.5^{\circ}$, which can be observed in Figs. 14 and 15 .

\section{- Separation and reattachment lines}

As discussed above, the gray-masked stripes visible in Figs. 13 and 14 at approximately $x / c<30 \%$ (data series S1 and S2), which are essentially oriented in the spanwise direction, were very likely related to the zero skin friction at the critical lines and to the large skin friction upstream and downstream of flow reattachment. In particular, this latter effect was expected to be the reason for the efficient oil-film removal around the reattachment location, which most probably led to the very low oil-film thickness in the span-wise-oriented stripes (see Fig. 12), and thus to the impossibility to estimate the skin friction using the GLOFSFE. The appearance of multiple stripes and the "smearing" of the gray-masked stripes with varying angle of attack in the same data series were also influences of the aforementioned time history of the oil film, but are discussed here because of their specificity. In fact, in series $\mathrm{S} 1$, the stripes related to the critical lines started to appear in Figs. 12, 13 and 14 from the data point S1-04 $\left(\mathrm{AoA}=0.8^{\circ}\right)$, and the number of stripes increased with increasing angle of attack up to $\mathrm{AoA}=1.5^{\circ}$ (data point S1-06). In practice, the stripes observed for a certain data point remained "imprinted" also in the subsequent data points. The approximate chord-wise locations of the stripes were in agreement with the expectations for the chord-wise locations of the critical lines, based on the measured pressure distributions (see Fig. 4). It should be noted here that, at $0.8^{\circ} \leq \mathrm{AoA} \leq 1.5^{\circ}$, the reattachment location expected for a certain angle of attack approximately overlapped the separation location expected for the subsequent, larger $\mathrm{A} o A$. It is therefore difficult to unequivocally discern between stripes related to flow separation or reattachment in the presence of multiple stripes; however, for the reasons discussed above, these most probably originated from the efficient oil-film removal around the reattachment location. The gray-masked stripes observed in series S2 appear to confirm this conjecture. In this second data series, a marked low- $\bar{h}$ /graymasked stripe was seen at $x / c \sim 19 \%$, starting from the first data point of the series (S2-01). An additional stripe was observed at $x / c \sim 22 \%$, starting from the data point S2-02. The chord-wise locations of these two stripes were in agreement with the expected reattachment locations at $\mathrm{AoA}=1.2^{\circ}$ and $\mathrm{AoA}=1.5^{\circ}$. In both data series, as the angle of attack was further increased, the low $-\bar{h} /$ gray-masked stripes were found to remain "imprinted" also in the subsequent data points, i.e., S1-07 and S1-08 in series $\mathrm{S} 1$, and $\mathrm{S} 2-03$ and $\mathrm{S} 2-04$ in series $\mathrm{S} 2$.

In series $\mathrm{S} 1$, as the angle of attack was reduced again, the stripes were observed to "smear": this is especially visible for the gray-masked regions of the data points S1-11 to S1-15. The different behavior observed for increasing and decreasing angle of attack was very likely related to the location of flow reattachment at a certain data point, with respect to that at the preceding data point. As the angle of attack was increased, flow separation and reattachment occurred at a more downstream location; because of the still low skin friction in the region around the previous location of flow reattachment, the "imprinted" distributions remained essentially unaffected by the change in AoA. In contrast, as the angle of attack was reduced, flow separation and reattachment occurred at a more upstream location; the now larger, positive skin friction in the region around the previous location of flow reattachment was capable of modifying the "imprinted" distributions, thus leading to the "smearing" of the low- $\bar{h}$ /gray-masked regions.

Because of these issues, it was not feasible to extract the locations of flow separation and reattachment from the gray-masked stripes in the GLOFSFE estimations.

\section{- Influence of the base coat}

The interaction between the oil film and the base coat applied to the measurement surface affected the GLOF measurements. One result of this interaction can be seen for series S1 in Fig. 12, where fixed cyan circular traces appeared at $y / c \sim 0.4$ and 0.6. These were traces of the oil droplets used in the calibration process (see Sect. 4.2), which can be observed in the oil-droplet image of Fig. 10c. The observed pattern was the result of the absorption of the remaining luminescent oil droplets by the base coat, and corresponded to approximately $3 \mu \mathrm{m}$ offset error in the oil-film thickness. The oil-droplet calibration was not repeated after the base coat had been changed (i.e., from series $\mathrm{S} 2$, see Sect. 4.3). Therefore, the oil patterns did not appear in series S2-S4.

Another result of the interaction between the oil film and the base coat can be seen in Fig. 12 for series S3 and $\mathrm{S} 4$. In these two latter data series, the oil had not been applied upstream of $x / c \sim 20 \%$, but appreciable luminescent intensity was still measurable even at $x / c<18 \%$. This luminescent intensity was due to the dye absorbed in this region during the preceding series $\mathrm{S} 2$. In this region, the corresponding offset error in the oil-film thickness of series S3 and S4 was approximately $5 \mu \mathrm{m}$. 
The offset error affects the estimation of the normalized skin-friction field (see Eqs. (5) and (6) in Sect. 2.1) as:

$$
\begin{aligned}
\mathbf{C} & =\frac{1}{4} \sum_{k}\left\{\operatorname{diag}\left(\mathbf{M}_{\mathrm{c} 2 \mathrm{f}}\left(\underline{\boldsymbol{r}}_{k}+\epsilon_{r}\right)\right)\right\}^{2} \boldsymbol{\Delta}_{\mathbf{x}}^{\mathrm{T}} \boldsymbol{\Delta}_{\mathbf{x}} \\
& \left\{\operatorname{diag}\left(\mathbf{M}_{\mathrm{c} 2 \mathrm{f}}\left(\underline{\boldsymbol{r}}_{k}+\epsilon_{r}\right)\right)\right\}^{2},
\end{aligned}
$$

and

$\mathbf{d}=-\frac{1}{2} \sum_{k}\left\{\operatorname{diag}\left(\mathbf{M}_{\mathrm{c} 2 \mathrm{f}}\left(\underline{\boldsymbol{r}}_{-k}+\epsilon_{r}\right)\right)\right\}^{2} \boldsymbol{\Delta}_{\mathbf{x}}^{\mathrm{T}} \boldsymbol{\Delta}_{\mathbf{t}}\left(\underline{\boldsymbol{r}}_{k}+\epsilon_{r}\right)$.

In the above equations, the offset error $\epsilon_{r}$ (assumed as a constant) is added to the ratioed image $\underline{\boldsymbol{r}}_{k}$.

This leads to an offset-induced error $\boldsymbol{\epsilon}_{\hat{\boldsymbol{\tau}}}$ in the estimated skin friction (see Eq. (3)) that can be presented as:

$\hat{\boldsymbol{\tau}}+\epsilon_{\hat{\tau}}=\tau_{*}\left(\hat{\tilde{\tau}}+\epsilon_{\hat{\tilde{\tau}}}\right)$,

where $\epsilon_{\hat{\tilde{\tau}}}$ is the offset-induced error in the normalized skin friction. In general, the relation between $\epsilon_{\tilde{\tilde{\tau}}}$ and $\epsilon_{r}$ is non-linear.

The offset error is particularly critical when the oil film is thin. As discussed in the beginning of this Subsection, the regions with low values of the coefficient of determination in Fig. 13 correspond to the areas with low oil-film thickness. These low values of $R^{2}$ were induced by the low signal-to-noise ratio of the GLOF images in these regions and by the offset error. In the present work, the offset error could not be compensated, because the pigmentation was neither uniform nor time-independent. The conduction of the GLOF measurements with a comparably thick oil film enabled the impact of the offset error in the GLOFSFE estimations to be mitigated; at the same time, the higher oil-film thickness induced the mutual interactions between the oil film and the boundary layer discussed above.

\section{- Turbulent wedges}

Turbulent wedges were observed superimposed on the quasi-2D skin-friction distributions for some of the data points (see Fig. 14). In the case of the data points S2-03 and S2-04, a large turbulent wedge was seen in the region around $y / c=0.60$. After the wind tunnel had been stopped, a particle was found on the oil film during model inspection. The position of the particle corresponded to the location of the apex of the wedge visible in Fig. 9b. Particles passing through the wind tunnel may be captured by the oil film and act as 3D roughness elements. A roughness element of critical size at a critical position could induce a three-dimensional flow and trigger bypass transition (see Sect. 2.3.1), as it was the case for the aforementioned particle in series $\mathrm{S} 2$. The small turbulent wedge observed in the data points S1-02 to S1-06 (around $y / c=0.57$ ) probably occurred in a similar manner.

The origin of the turbulent wedge seen in the data points of series S4 (S4-03 to S4-06) around $y / c=0.51$ was different. The span-wise location of the wedge apex matched that of a channel for the installation of the tubes for the pressure taps, which was filled by resin before the experiments were started (see Fig. 1). However, the filling resin contracted over time and formed a shallow depression, which also acted as a 3D surface imperfection and induced the occurrence of the turbulent wedge seen in series S4. Interestingly, a small turbulent wedge at approximately the same span-wise location was observed also in the initial data points of series S1 and S3 (i.e., S1-01 and S3-01). It is possible that, because of the presence of the oil film on the model surface, the boundary layer had not reached stable conditions during the acquisition of the initial data points of these two series; the otherwise small three-dimensional disturbances originating from the filled channel may have had larger initial amplitude or may have undergone stronger amplification, as compared to the other data points, thus leading to the formation of a turbulent wedge at a more downstream location.

The sources of the further turbulent wedges observed in Fig. 14 at the span-wise ends of the evaluation region (data points $\mathrm{S} 1-07$ to S1-08, S3-01 to S3-02, S3-05, and S4-03 to S4-04) were the edges of the base coat. These turbulent wedges can be also seen in the oil-film patterns shown in Figs. 2 and 9b. The base-coat edges were span-wise steps of approximately $100 \mu \mathrm{m}$ height, running from the leading edge to the trailing edge in the streamwise direction. The step height at the leading edge was sufficiently large so as to affect the laminar flow (see Sect. 4.1.1), and bypass transition occurred.

As discussed in the beginning of this subsection, the GLOFSFE estimations in the turbulent-flow regions can be considered only as qualitative. They can still be meaningful for the examination of the topology of the skin-friction distribution, in a manner similar to that of conventional oilflow visualizations: the identification of turbulent wedges in Fig. 14, as presented above, is an example of such qualitative analysis. However, the turbulent skin-friction estimations of the current study cannot be analyzed in quantitative terms. This includes the challenge in clearly and reliably identifying the location corresponding to the end of the transition region, as defined in Sect. 4.1.1: the location where $C_{f}$ reached $C_{f, \mathrm{~T}}=3 \cdot 10^{-3}$. An alternative to this definition may have been the location where $C_{f}$ reached its maximum, after the transition process had started. Because of the inaccurate 
skin-friction estimations downstream of the location of transition onset, however, a maximum in the skin-friction distribution could not be reliably identified. This can be seen in Fig. 15, where a distinct peak in the skin-friction coefficient downstream of the location of transition onset was not detectable for various data points, as due to the issues discussed above.

In contrast, a quantitative analysis of the skin-friction estimations in the laminar-flow regions was feasible for some of the examined data points, since the oil-film thickness was below the critical limit of surface roughness. At the same time, the uncertainty in the skin-friction values estimated in these areas was relatively high, as indicated by the low value of the coefficient of determination $R^{2}$. As a first step of this analysis, the distributions of the average oil-film thickness and of the skin-friction coefficient were surveyed to identify the data points with reliable estimations in the laminar-flow regions. By consideration of the issues discussed above, the following data points were discarded:

- S1-01 to S1-03, S2-03 to S2-04, and S3-01 to S3-02, because of the premature transition induced by the oil 'bump';

- S2-01 to S2-02, because of the 'bump' in the skin-friction distribution induced by the oil 'bump' upstream of the natural transition onset;

- S3-03 to S3-04, and S4-01 to S4-02, because the streamwise extent of the laminar-flow region measurable via GLOF was only a few percent of the chord, before the (shock-induced) transition process was initiated. (The luminescent oil was applied at $x / c \sim 20 \%$ prior to series S3.)

The chord-wise profiles of the skin-friction coefficient estimated for the remaining data points were examined. As described for Fig. 15, these are the averages of the $C_{f x}$ distributions in the laminar regions where the flow was essentially two-dimensional; accordingly, the areas affected by turbulent wedges were excluded. The $C_{f x}$ profiles were analyzed to extract the standard deviation (or the difference, when only two data points were available) of the estimated skinfriction coefficients in evaluable parts of the laminar-flow regions, i.e., where $C_{f x}$ profiles were obtained for different data points over the same portion of the chord. Representative uncertainties $\Delta C_{f x}$ for the estimations of the skin-friction coefficient were then determined as stream-wise averages of the extracted distributions of the $C_{f x}$ standard deviation (or difference) over these portions of the chord. The obtained, representative uncertainties ranged from approximately $\Delta C_{f x}=3 \cdot 10^{-5}$ to $1.5 \cdot 10^{-4}$. These values correspond to approximately $\pm 8 \%$ to $\pm 38 \%$ of the reference laminar skinfriction coefficient $C_{f, \mathrm{~L}}$. As already mentioned in Sect. 5.4, a full uncertainty analysis, such that conducted in Lee et al. (2020c), could not be carried out in the present work. This was due not only to the missing information on the offset error in the oil-film thickness estimation (see above), but also to the variation of the local flow conditions along the stream-wise direction over the examined airfoil surface. Furthermore, the oil-film thickness distributions for the evaluable data points were different even for the same angle of attack (see Fig. 12). As a conservative estimation, the maximal value $\Delta C_{f x}=1.5 \cdot 10^{-4}$ from the aforementioned analysis based on the $C_{f x}$ profiles was taken as a representative uncertainty of the skin-friction coefficient distributions in the laminar-flow regions. This value appears reasonable as a conservative estimation, since it would also cover the contributions of all uncertainties in the GLOFSFE parameters described in Sect. 5.4.

The location of transition onset $x_{\mathrm{T}, \text { onset }} / c$ could be also determined quantitatively for the evaluable data points identified in the aforementioned analysis. Additionally, also the data points S3-03 to S3-04 and S4-01 to S4-02 could be considered for the evaluation of $x_{\mathrm{T} \text {,onset }} / c$. In contrast to the previous analysis, for this scope, the short stream-wise extent of the measurable laminar-flow region was sufficient to detect the increase in $C_{f x}$ related to the initiation of the transition process. As introduced in Sect. 4.1.1, transition onset was detected at the location where $C_{f}$ became larger than $C_{f, \mathrm{~L}}=4 \cdot 10^{-4}$. In Fig. 15 , it can be seen that the locations of transition onset detected for the evaluable data points were in reasonable agreement, with a maximal deviation of $\Delta x_{\mathrm{T} \text {,onset }} / c=5 \%$ at AoA $=0.8^{\circ}$. In most of the other cases, the deviation was $\Delta x_{\mathrm{T} \text {,onset }} / c=3 \%$ or less.

The GLOFSFE estimations in the laminar-flow regions and the detected locations of transition onset will be further discussed in Sect. 6.3, where a comparison with the transition locations measured via TSP in recent experiments (Costantini et al. 2020) will be also presented.

As a final consideration in this subsection, it should be remarked that the CFL number was sufficiently low even in the turbulent-flow regions. As a representative example, the CFL number (calculated from Eq. (13)) in the turbulent-flow region was approximately 2.7 at $x / c=30 \%$ for the data point $\mathrm{S} 2-02$. The coefficient of determination at this location was $R^{2}=0.53$, so that the numerical process appeared to be valid even when the CFL number exceeded unity. The maximally allowable $C F L$ number $C F L_{\max }$ could be larger than one, because the oil-film distribution has a continuous length.

\subsection{Aerodynamic coefficients}

The aerodynamic coefficients estimated for all examined data points are presented in Fig. 16. For the reasons discussed in subsection 3.1, the values of the aerodynamic coefficients should be regarded only as qualitative. With 
Fig. 16 Lift, drag, and pitching moment coefficients on the airfoil model as measured by the pressure distribution and the wake deficit at $M=0.72$ and $R e_{c}=10^{7}$
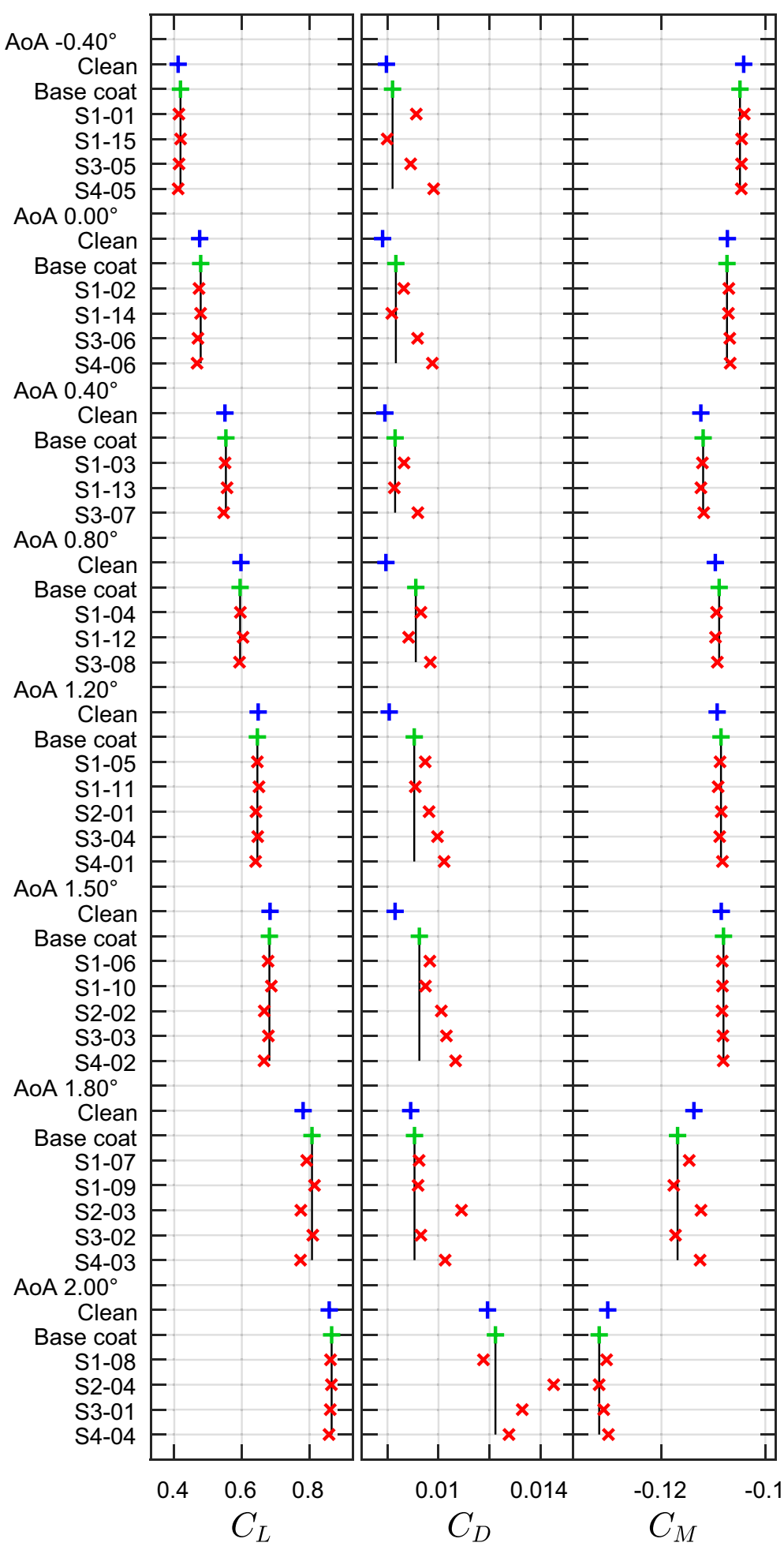

the base coat applied, the spatial resolution of the measured pressure distribution, which served for the estimation of the lift coefficient $C_{L}$ and of the pitching moment coefficient $C_{M}$, was even further reduced, as compared to the clean configuration. In fact, the pressure taps on the lower side of the leading-edge area were covered by the 
base coat and were thus unavailable; additionally, $C_{L}$ and $C_{M}$ had to be calculated from the starboard-side pressure distribution (at $y / c=0.68$ ), where the number of pressure taps was less than that of the central pressure tap row. Nevertheless, the estimated values of $C_{L}, C_{M}$, and of the drag coefficient $C_{D}$ could be considered for the evaluation of relative changes in the aerodynamic coefficients due to the oil-film setup. This included not only the global influence of the oil film, but also the effect of the base coat, which was surveyed by comparing the aerodynamic coefficients estimated for the clean configuration (i.e., without the base coat) with those of the base-coat only cases (i.e., without the oil film).

The estimated $C_{L}$ and $C_{M}$ were in agreement for all the examined cases despite the presence of the base coat and of the oil film (deviations in the lift and pitching moment coefficients within $\Delta C_{L}= \pm 2.5 \%$ and $\Delta C_{M}= \pm 1 \%$ from the clean configuration values), except for the case at AoA $=1.8^{\circ}$, at which the maximal $\Delta C_{L}$ and $\Delta C_{M}$ were approximately $\pm 4 \%$ of the clean configuration values. This case was found to be particularly sensitive to even very small variations (within the parameter accuracy presented in Sect. 3.1) in the Mach number, in the flow total temperature, and in the wind-tunnel wall contour; for this reason, it resulted difficult to reproduce the shock-wave position, as confirmed by the Schlieren images.

A global effect of the oil-film setup can be seen in the variation of the drag coefficient. Comparing the drag coefficient estimated for the clean configuration with that of the base-coat only cases, the presence of the base coat was found to already lead to a significant increase in $C_{D}$ for most of the examined data points. This was very likely due to the generation of turbulent wedges from the span-wise steps at the base-coat edges (see Sect. 6.1), but also the change in airfoil contour due to the application of the base coat may have contributed to the $C_{D}$ increase.

In the presence of the oil film, the larger additional increases in the drag coefficient were found for the data points with large turbulent wedges (e.g., S2-03, S2-04, S4-05, and S4-06, see Figs. 14 and 16). Moreover, the trend observed in Fig. 16 is that data points with thinner oil film corresponded to smaller additional increases in $C_{D}$. This can be seen, for example, by a comparison of S1-01 with S1-15, S1-02 with S1-14, etc. In fact, the first data points of series S1 were also affected by the oil 'bump', which led to premature transition (see Sect. 6.1). When the oil film is thin, the additional increase in the drag coefficient is essentially negligible, as shown, e.g., by the comparison of the base-coat only cases with the data points S1-07 to S1-15. The general trend of larger additional $C_{D}$ increases with larger oil-film thickness supports the discussion of the oil-film influence on the estimated skin-friction distributions, which was presented in the previous subsection. However, it should be emphasized that the base coat already led to significant increases in the drag coefficient: in particular, at $0.8^{\circ} \leq \mathrm{AoA} \leq 1.5^{\circ}$ the effect of the base coat on the wake deficit was even larger than that due to the oil film.

\subsection{Discussion of the GLOFSFE estimations in the laminar-flow region and of the detected locations of transition onset}

The evaluable data points from the analysis presented in Sect. 6.1 were further surveyed to select, for each angle of attack, a single data point with a higher confidence level in the laminar-flow regions. For this reason, the data points S1-09 to S1-15 were discarded, since the coefficient of determination $R^{2}$ was smaller than that of the data points $\mathrm{S} 1-04$ to S1-07 and S3-05 to S3-07. Moreover, the data points S3-05 and S3-06 were preferred to the corresponding data points from series S4 (S4-06 and S4-05, respectively), because these latter data points were affected by a large turbulent wedge around $y / c=0.51$.

The $C_{f x}$ distributions of the selected data points are shown in Fig. 17. The profiles of $C_{f x}$ and $\bar{h}$ along with the reference values $C_{f, \mathrm{~L}}, C_{f, \mathrm{~T}}$, and the estimated viscous sublayer thickness $\delta_{\mathrm{v}}=5 \mu_{\text {air }} / \sqrt{\tau \rho_{\text {air }}}$ (see Eq. (21)) are shown in Fig. 17a. The corresponding $C_{f x}$ distributions from Fig. 14 are shown in Fig. 17b. In Fig. 17a, it can be seen that noisy distributions of $C_{f x}$ and $\bar{h}$ corresponded to the low $h$ regions, approximately where $\bar{h}^{+}<3$. Interestingly, it appears that the quality of the GLOFSFE estimations in the laminarflow regions improved as $\bar{h}^{+}$approached $\bar{h}^{+} \sim 5$, in a manner similar to the observations of Lee et al. (2020c) for a turbulent flat-plate boundary layer. However, larger values of $\bar{h}^{+}$(approximately $\bar{h}^{+}>7$ ) in the laminar-flow region close to the leading edge led to overestimations of the skin-friction coefficient, as will be shown below. These overestimations were observed for the data points S1-04 to S1-08, where the oil film was thick in the GLOFSFE-evaluation region close to the leading edge because of the oil 'bump' affecting these data points (see Fig. 12 and the related discussion in Sect. 6.1).

To obtain reference skin-friction values in the laminarflow region, compressible boundary-layer calculations were carried out using the laminar boundary-layer solver COCO (Schrauf 1998), which is a well established code for boundary-layer stability analysis and laminar wing design (see Streit et al. (2015) and Schrauf and von Geyr (2020), among others). The surface pressure distributions, measured by means of the pressure taps, served as inputs for the boundary-layer computations, together with the freestream Mach number, the chord Reynolds number, and 
Fig. 17 Chord-wise skinfriction coefficient profiles at $M=0.72$ and $R e_{c}=10^{7}$ for selected data points. a Estimated skin-friction coefficient $C_{f x}$ (dash-dotted blue lines), numerically computed laminar skin-friction coefficient $C_{f, \text { num }}$ (solid green lines), and oil-film thickness $\overline{h^{+}}$(dashed red lines) averaged along the span. b Corresponding skin-friction distributions. The gray areas indicate the masked areas due to missing image data or ill-posed nodes

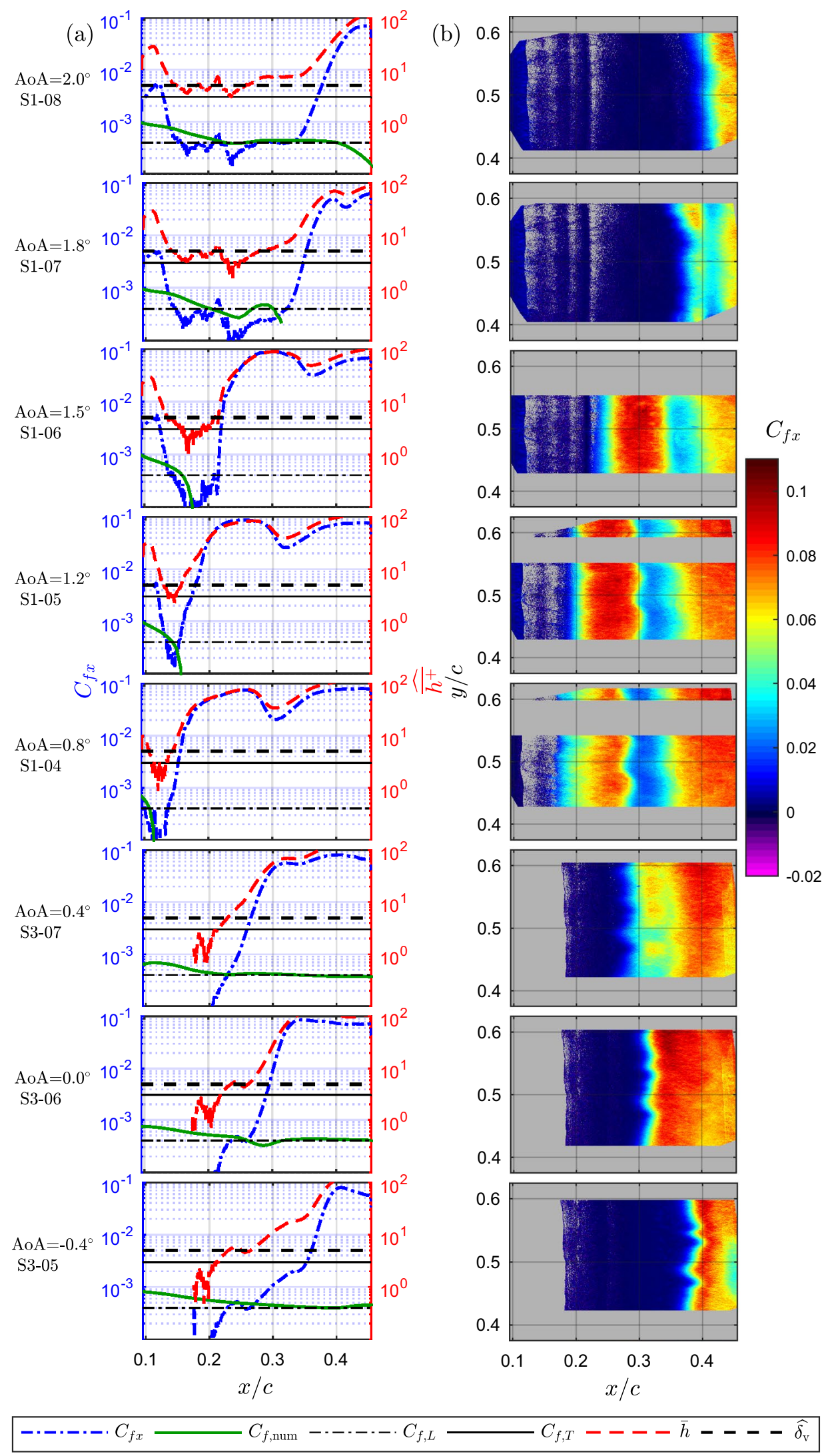


the freestream static temperature. The model surface was reasonably assumed to be an adiabatic wall. The streamwise distributions of the laminar friction coefficient computed via $\mathrm{COCO}$ for the examined angles of attack are also shown in Fig. 17 a $\left(C_{f, \text { num }}\right)$. Moreover, zoomed-in plots of the comparisons between the profiles of $C_{f x}$ estimated via GLOFSFE and those computed using COCO are presented in Fig. 18. In this latter figure, only the chord-wise region up to $x / c=40 \%$ and the $C_{f x}$ values between $10^{-4}$ and $10^{-3}$ are shown in the horizontal and vertical axes, respectively. The reference value for the laminar skin-friction coefficient $C_{f, \mathrm{~L}}$ with the representative uncertainty estimated in Sect. 6.1 are also presented in Fig. 18. It should be emphasized here that the numerical computations, performed with a laminar boundary-layer solver, could not take into account the transition process; they therefore provide skin-friction data that are meaningful for comparison only in the region upstream of the location corresponding to the transition onset, as observed in the experiments. Moreover, COCO predicted laminar separation to occur in the examined region for $0.8^{\circ} \leq \mathrm{AoA} \leq 1.8^{\circ}$; for this reason, no numerical data are available downstream of $x_{\mathrm{S}} / c \sim 12 \%, 16 \%, 18 \%$, and $31 \%$ for the angles of attack AoA $=0.8^{\circ}, 1.2^{\circ}, 1.5^{\circ}$, and $1.8^{\circ}$, respectively. In Figs. 17a and 18, it can be seen that the value assumed in Sect. 4.1.1 for the reference laminar skin-friction coefficient $C_{f, \mathrm{~L}}=4 \cdot 10^{-4}$ was, in general, representative for the computed laminar $C_{f}$. This was observed for a significant chord-wise portion of the investigated airfoil region in the majority of the examined cases, except for the cases at AoA $=0.8^{\circ}, 1.2^{\circ}$, and $1.5^{\circ}$, where laminar separation was predicted close to the leading-edge area (i.e., at $x / c<20 \%$ ).

As introduced in Sect. 1, reference transition locations have become available via recent experiments (Costantini et al. 2020), in which the VA-2 airfoil model was equipped with a TSP and investigated for the same test cases as those examined in the present work. The measured surface temperature distributions were analyzed along five evaluation sections, which were located at different span-wise positions. For each evaluation section, transition was detected at the location corresponding to the maximal temperature gradient in the stream-wise direction, according to the procedure described in Costantini (2016). The transition locations $x_{\mathrm{T}} / c$ and the related uncertainties $\Delta x_{\mathrm{T}} / c$ reported for the TSP data from Costantini et al. (2020) are the averages and the standard deviations of the transition locations detected at the five evaluation sections. Because of the difference in the definition of $x_{\mathrm{T}, \text { onset }} / c$ and $x_{\mathrm{T}} / c$, the transition location from the TSP data analysis is expected to be always more downstream than the location of transition onset estimated via GLOFSFE. Nevertheless, the distance between these two locations may be small when transition is induced by a strong adverse pressure gradient (see Costantini (2016)). A comparison of the locations of transition onset estimated via
GLOFSFE with the transition locations measured via TSP is presented in Fig. 19. Note here that the reported values of $\Delta x_{\mathrm{T}, \text { onset }} / c$ correspond to the maximal deviation of the locations of transition onset estimated via GLOFSFE for the evaluable data points at a certain AoA (see Sect. 6.1), i.e., it is an uncertainty of a different nature than that reported for the TSP results.

With consideration of the representative measurement uncertainty, the comparisons in Figs. 17a and 18 show, in portions of the chord for some of the examined data points, reasonable agreement of GLOFSFE estimations and numerical results. Moreover, the comparison of the transition locations presented in Fig. 19, considering also the reported experimental deviations and uncertainties, shows significant agreement of the GLOFSFE estimations with the TSP results for most of the investigated angles of attack.

- At AoA $=1.8^{\circ}$ and $2.0^{\circ}$, the reasonable agreement of the estimated and computed $C_{f x}$ profiles was observed in a significant stream-wise region between $x / c \sim 18 \%$ and the location corresponding to the transition onset in the experiments, except for a small region around $x / c \sim 24 \%$, where a minimum of the estimated $C_{f x}$ can be seen. The low values of estimated $C_{f x}$ in this small region were due to the locally thinner oil film, as compared to the upstream and downstream regions (see Figs. 12 and 17a). The locally thinner oil film was probably induced by the influences of the time history of the oil film and of the presence of critical lines in the preceding data points (see Sect. 6.1); in particular, the low values of estimated $C_{f x}$ in the region around $x / c \sim 24 \%$ were likely related to the oil 'valley' formed from data point S1-03 and to the region of apparently nearly-zero skin friction formed from data point S1-06.

In the remaining portions of the aforementioned stream-wise region of reasonable agreement, the numerical predictions were within the $C_{f x}$ range given by the GLOFSFE estimations with the representative measurement uncertainty. Moreover, at AoA $=1.8^{\circ}$, the estimated location of transition onset was very close to the predicted location of laminar separation $\left(x_{\text {T.onset }} / c\right.$ was indeed slightly downstream of $x_{\mathrm{S}} / c \sim 31 \%$ ). At these flow conditions, transition was expected to start shortly downstream of the boundary-layer separation induced by the adverse pressure gradient at $x / c>30 \%$ (see Fig. 4). The estimated location of transition onset was also slightly upstream of the transition location from the TSP experiments, as can be seen in Fig. 19. The observed agreement of the locations of predicted laminar separation, estimated transition onset, and transition detected via TSP was a notable result. At AoA $=2.0^{\circ}$, transition occurred in a region significantly upstream of the location of the shock visible in Fig. 4. It appears that the 

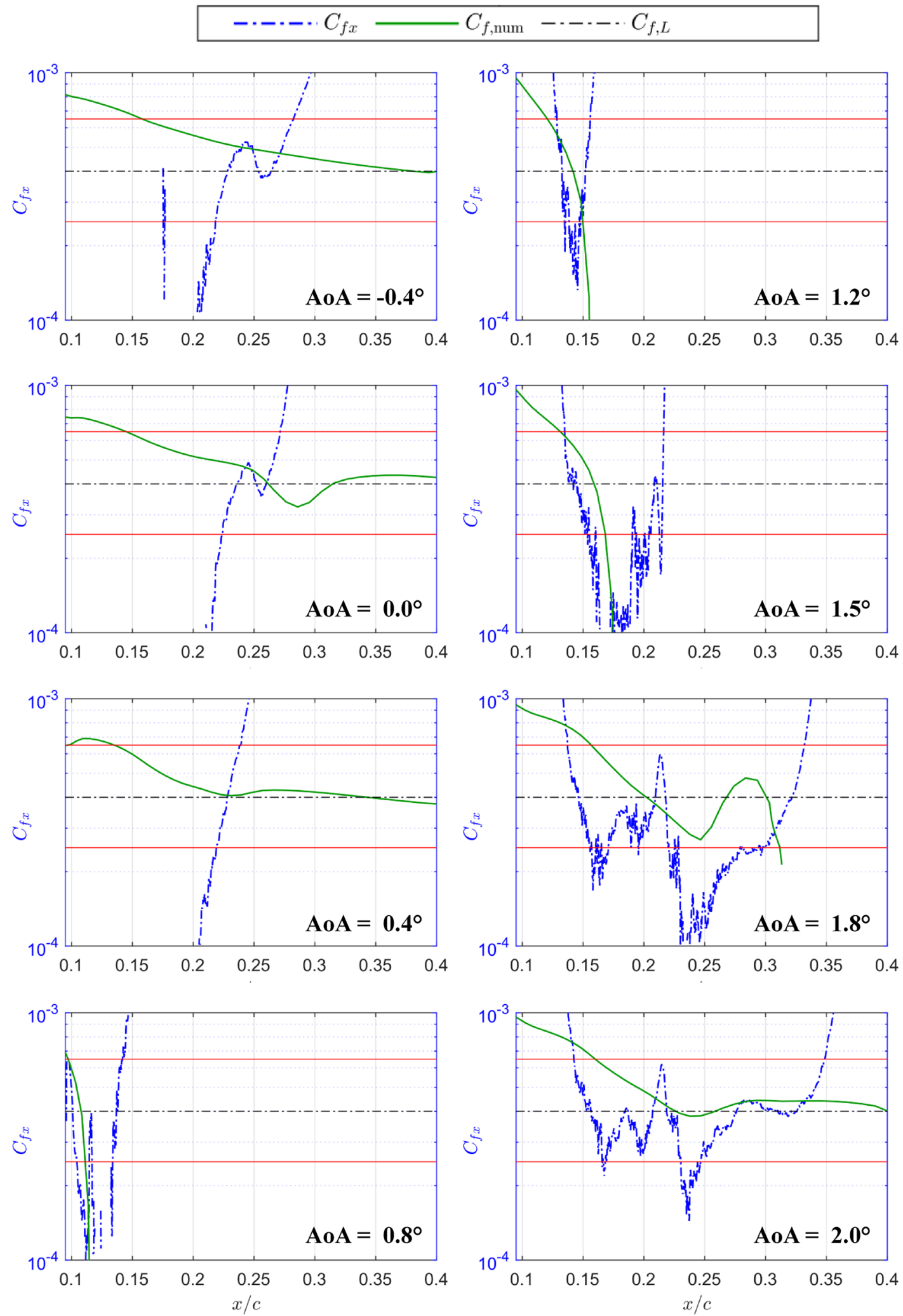

Fig. 18 Zoomed-in plots of the skin-friction coefficient profiles at $M=0.72$ and $R e_{c}=10^{7}$ presented in Fig. 17. Comparison of the estimated skin-friction coefficient $C_{f x}$ (dash-dotted blue lines) with the numerically computed laminar skin-friction coefficient $C_{f \text {,num }}$ (solid

green lines). The reference value for the laminar skin-friction coefficient $C_{f, \mathrm{~L}}$ (dash-dotted black line) with the estimated, representative uncertainty (red lines) is also shown 
adverse pressure gradient in the region at approximately $30 \% \leq x / c \leq 37 \%$ led to marked amplification of the boundary-layer disturbances and thus induced transition, even though the laminar boundary layer was not predicted to separate (see Figs. 17a and 18). The location of transition onset estimated via GLOFSFE was found to be upstream of the transition location measured via TSP, with a small distance between these two locations, in agreement with the expectations.

At approximately $x / c<18 \%$, the values of skinfriction coefficient obtained for both angles of attack via GLOFSFE were larger than the predicted $C_{f x}$, since the oil film was too thick in this region close to the leading edge (see Fig. 12). As can be seen in Fig. $17 \mathrm{a}, \bar{h}^{+}>7$ seems to provide an indication for the regions where the skin-friction coefficient was overestimated.

- At $0.8^{\circ} \leq$ AoA $\leq 1.5^{\circ}$, the agreement of the computed and estimated $C_{f x}$ profiles (considering also the representative measurement uncertainty) was reasonable for small stream-wise regions up to the predicted location of laminar separation: from $x / c \sim 9 \%$ to $x_{\mathrm{S}} / c \sim 12 \%$ at $\mathrm{AoA}=0.8^{\circ}$, from $x / c \sim 13 \%$ to $x_{\mathrm{S}} / c \sim 16 \%$ at $\mathrm{AoA}=1.2^{\circ}$, and from $x / c \sim 13 \%$ to $x_{\mathrm{S}} / c \sim 18 \%$ at $\mathrm{AoA}=1.5^{\circ}$. Similarly to the cases presented above, $C_{f x}$ at $\mathrm{AoA}=1.2^{\circ}$ and $1.5^{\circ}$ was overestimated upstream of $x / c \sim 13 \%$, i.e., in the region close to the leading edge where the oil film was too thick. As compared to AoA $=1.8^{\circ}$ and $2.0^{\circ}$, however, the overestimation region was of smaller stream-wise extent for these lower angles of attack; in particular, at AoA $=0.8^{\circ}, \overline{h^{+}}$remained close to or below 5 for the whole GLOFSFE-evaluation region up to the estimated location of transition onset. This difference with the two cases at the largest AoA was due to the local increase in the boundary-layer thickness at approximately $x / c>9 \%$ induced by the adverse pressure gradient observed in this region for $0.8^{\circ} \leq \mathrm{AoA} \leq 1.5^{\circ}$, as compared to the nearly-zero pressure gradient for AoA $=1.8^{\circ}$ and $2.0^{\circ}$ (see Fig. 4). Also in the three cases at $\mathrm{AoA}=0.8^{\circ}, 1.2^{\circ}$, and $1.5^{\circ}$, boundary-layer transition was expected to start shortly downstream of the laminar separation induced by the strong adverse pressure gradient (very marked in the case of the shock wave at AoA $=1.2^{\circ}$ and $1.5^{\circ}$ ). The estimated location of transition onset was slightly upstream of the predicted location of laminar separation at AoA $=1.2^{\circ}$, whereas it was slightly downstream at $\mathrm{AoA}=0.8^{\circ}$ and $1.5^{\circ}$. Considering also the reported deviations $\Delta x_{\mathrm{T} \text {,onset }} / c$ in the GLOFSFE estimations, the results were in agreement with the expectations. Moreover, the locations of transition onset estimated via GLOFSFE were in agreement with the transition locations measured via TSP (see Fig. 19).

- At AoA $\leq 0.4^{\circ}$, the comparison of computed and estimated $C_{f x}$ profiles for the selected data points was pos- sible only for a laminar-flow region of small stream-wise extent, since the GLOFSFE estimations for the data points S3-05 to S3-07 were available at approximately $x / c \geq 20 \%$. With consideration of the representative measurement uncertainty, the numerical and estimated $C_{f x}$ values at $\mathrm{AoA}=0.0^{\circ}$ and $-0.4^{\circ}$ were in reasonable agreement in the small stream-wise regions between $x / c \sim 0.22$ and the estimated location of transition onset, i.e., for approximately $\Delta x / c=4-5 \%$. The comparison for the case at $\mathrm{AoA}=0.4^{\circ}$ is presented here only for the sake of completeness, since transition was observed to start in the experiments at an even more upstream location $\left(x_{\mathrm{T} \text {,onset }} / c \sim 23 \%\right)$ than that at $\mathrm{AoA}=0.0^{\circ}$ and $-0.4^{\circ}$. Therefore, reasonable agreement of numerical and estimated $C_{f x}$ values could be observed only in the proximity of the estimated location of transition onset.

At AoA $=-0.4^{\circ}$, the location of transition onset estimated via GLOFSFE was in agreement with the transition location measured via TSP (see Fig. 19). However, at $\mathrm{AoA}=0.0^{\circ}$ and $0.4^{\circ}, x_{\mathrm{T} \text {,onset }} / c$ was downstream of the location $x_{\mathrm{T}} / c$ detected in the TSP measurements. An analysis of the TSP results revealed that transition occurred prematurely at these two latter angles of attack, very likely because of an imperfection in the contour of the model when it was equipped with TSP. Therefore, the difference from the expectations observed at AoA $=0.0^{\circ}$ and $0.4^{\circ}$ seems to be ascribable to the TSP measurements, rather than to the GLOFSFE estimations.

Figure 20 shows the estimations of the transition regions and of the shock-wave positions for the selected data points with varying angle of attack. As discussed in Sect. 6.1, the reported values of $x_{\mathrm{T}, \text { onset }} / c$ (blue diamonds) are quantitative estimations obtained using the GLOFSFE, whereas the locations of transition end (blue squares) can be considered only as qualitative. The shock-wave positions were estimated from the measured surface pressure distributions at the center positions of the adverse pressure gradient interval, and the uncertainties (presented as green bars) were evaluated from the tap interval width. Note here that the starboard-side pressure taps were used in this case, since the central row of pressure taps was covered by the base coat (see Sect. 3.1). Therefore, the resolution of the pressure distribution was reduced, as compared to Fig. 4. The shockwave positions could be confirmed by the Schlieren images in the cases at $\mathrm{AoA}=1.8^{\circ}$ (see Fig. 11) and $\mathrm{AoA}=2.0^{\circ}$, since in these cases, the shock wave occurred within the available Schlieren windows (see Fig. 2 and Sect. 3.3). The reported shock-wave positions (magenta crosses) were determined by taking the location corresponding to the middle of the dark strip in the time-averaged Schlieren image, while their uncertainties (magenta bars) were evaluated from the variation of the dark strip location in the time series of the 


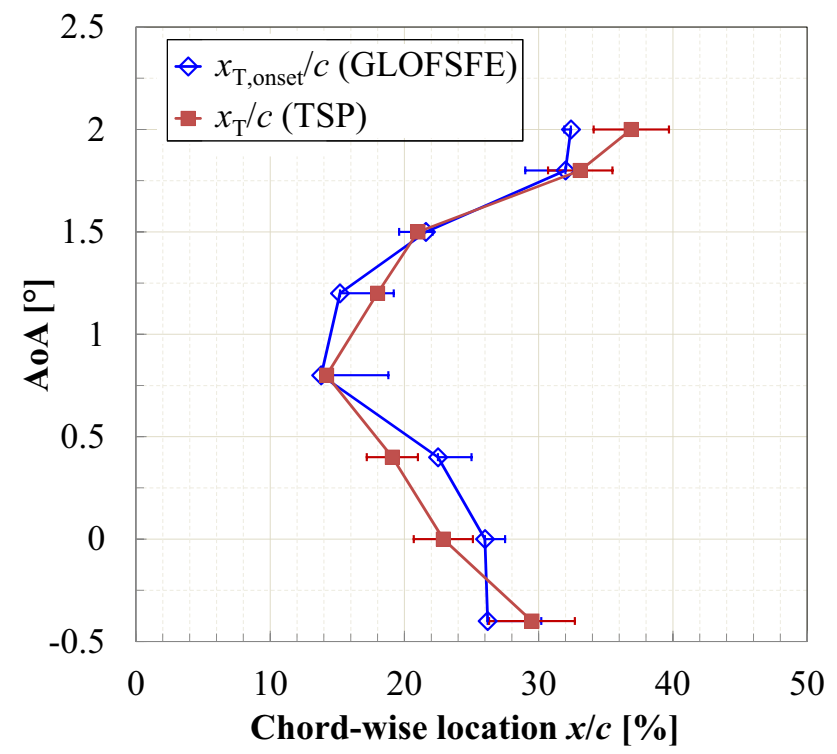

Fig. 19 Comparison of locations of transition onset estimated via GLOFSFE and transition locations measured via TSP (Costantini et al. 2020). The error bars correspond to the deviation of the GLOFSFE estimations $\Delta x_{\mathrm{T}, \text { onset }} / c$ and to the uncertainty of the TSP results $\Delta x_{\mathrm{T}} / c$

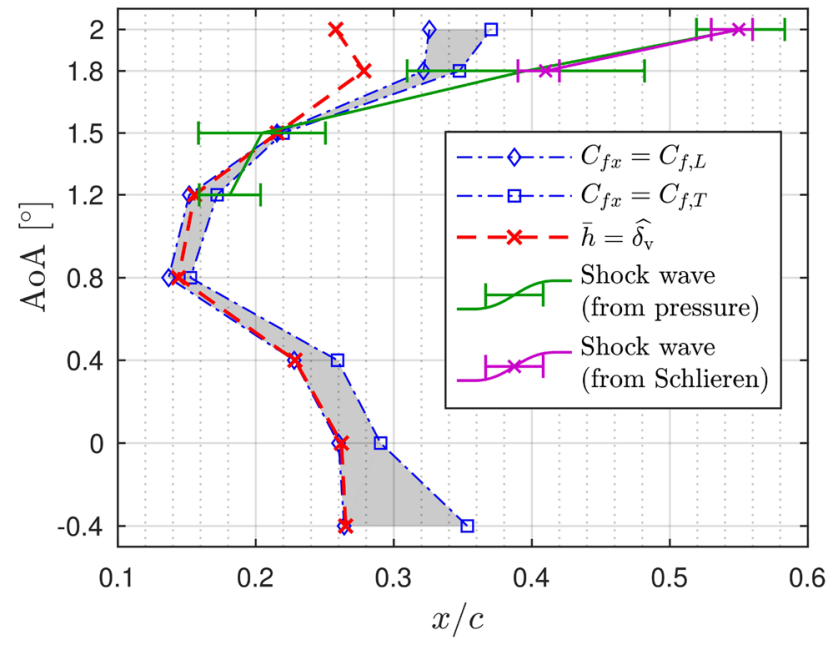

Fig. 20 Estimations of the transition regions and of the shock-wave positions for the selected data points with varying angle of attack. The locations where the estimated oil-film thickness matched the estimated viscous sublayer thickness are also shown

images. Moreover, the red crosses in Fig. 20 indicate the locations where the estimated oil-film thickness $\bar{h}$ matched the estimated viscous sublayer thickness $\delta_{\mathrm{v}}$.

As can be seen in Fig. 20, the estimated transition region was found to move upstream when the angle of attack was increased from $\mathrm{AoA}=-0.4^{\circ}$ to $0.8^{\circ}$. This evolution was induced by the more pronounced pressure minimum at $x / c \sim 8 \%$ and by the following stronger adverse pressure gradient (see Fig. 4). As discussed above, at AoA $=0.8^{\circ}$, transition occurred very likely within a laminar separation bubble, induced by the markedly adverse pressure gradient observed in this case. At larger angles of attack, shock waves were seen in the airfoil pressure distribution, and confirmed by the Schlieren images in the cases of AoA $=1.8^{\circ}$ and $2.0^{\circ}$. The downstream movement of the transition region with increasing angle of attack for AoA $\geq 1.2^{\circ}$ was related to the change in the pressure distribution at these transonic flow conditions. At AoA $=1.2^{\circ}$ and $1.5^{\circ}$, the shock-wave location matched that of the transition region: in these cases, boundary-layer transition was induced by the shock wave. In contrast, at $\mathrm{AoA}=1.8^{\circ}$ and $2.0^{\circ}$, transition was found to start at a location upstream of the shock-wave position. As discussed above, the adverse pressure gradient in the region at approximately $30 \% \leq x / c \leq 37 \%$ appeared to induce boundary-layer transition at the two largest angles of attack. The evolution of the transition region with varying angle of attack estimated via GLOFSFE was generally in agreement with the change in the transition location as a function of AoA measured via TSP (Costantini et al. 2020)—see Fig. 19.

Finally, it is interesting to note in Fig. 20 that $x\left(h=\delta_{\mathrm{v}}\right) \leq x\left(C_{f}=C_{f, L}\right)$ held in most of the cases, clearly indicating that the oil-film thickness in the turbulent-flow regions was larger than the critical limit of roughness.

\section{Conclusions and recommendations for future work}

The feasibility of global skin-friction measurements based on GLOF image data was examined in this work for the challenging flow conditions of a freestream Mach number of 0.72 and a chord Reynolds number of $10^{7}$. The experiments were performed in the Transonic Wind Tunnel Göttingen on the VA-2 supercritical airfoil model, focusing on the model upper surface. The model angle of attack was varied from $\mathrm{AoA}=-0.4^{\circ}$ to $2.0^{\circ}$, thus enabling the study of different boundary-layer stability situations: from a moderately favorable pressure gradient at the lowest AoA to cases with shock-wave/boundary-layer interaction at the larger angles of attack. The GLOF measurements were supported by conventional pressure measurements (on the surface and in the wake of the model) as well as by Schlieren flow visualizations. Since the optical access was available only from the test-section side walls, a camera calibration and image projection procedure was applied to obtain the GLOF results on the three-dimensional model surface. The skin-friction estimations were obtained from the GLOF image data according to the GLOFSFE, where an LLS-based approach was used for the GLOF image analysis.

An oil with high kinematic viscosity $(60,000 \mathrm{cSt}$ at a temperature of $25^{\circ} \mathrm{C}$, selected for the operating conditions 
of the continuously driven wind tunnel) was applied on a base coat, which covered the model surface. Different initial oil positions and distributions were considered to investigate the most appropriate starting conditions for the GLOF measurements, and different sequences of angles of attack were examined for each wind-tunnel operation series with a certain oil starting condition. The repeatability of the results was found to be limited by the influence of the initial oil position, by the effect of the oil-film time history, by turbulent wedges, and by the influence of the used base coat. Depending on the initial oil position and on the time history of the oil film, oil 'bumps' and 'valleys' formed on the model surface for some of the examined cases, and especially in the first data points of each operation series; they could lead to premature transition and/or to local under-/ overestimations of the skin-friction coefficient. Moreover, in the estimated skin-friction distributions at the higher angles of attack, the presence of laminar separation bubbles induced by the considered pressure distributions was very likely the reason for the appearance of regions that were detected as ill-posed by the GLOFSFE code. In fact, these regions appeared as stripes, essentially oriented in the spanwise direction, around the chord-wise locations corresponding to the expected locations of reattachment, i.e., where the large skin friction was expected to remove efficiently the luminescent oil, thus leading to the formation of stripes with very low GLOF signal. As the angle of attack was increased, these stripes were observed to remain "imprinted" also in the estimated oil-film thickness and skin-friction distributions of the subsequent data points. Turbulent wedges occurred for some of the examined data points, triggered by threedimensional imperfections such as the edges of the base coat or particles captured by the oil film during wind-tunnel operation. The used base coat absorbed luminescent dye, leading to an offset error in the oil-film thickness estimation. The influence of the offset error on the GLOF measurements could be mitigated by using a comparably thick oil film, but this made impossible to obtain quantitative skin-friction estimations in the turbulent-flow regions, since the oil-film thickness was larger than the viscous sublayer thickness and the surface was not hydraulically smooth. For this reason, the oil film interacted with the boundary layer, thus leading to large increases in the turbulent skin friction, as compared to the clean configuration.

In contrast, quantitative skin-friction estimations based on GLOF data were obtained, for the first time at the investigated test conditions, in the laminar-flow regions. In fact, for a group of the examined data points, the oil-film thickness was generally below the critical limit of roughness that would have led to premature transition. However, the thin oil film in the laminar-flow regions also resulted in a significant uncertainty in the skin-friction estimations. Noisy distributions of the estimated skin-friction coefficients were observed in the regions where the oil-film thickness in wall units $h^{+}$was approximately below 3 . The accuracy of the GLOFSFE estimations appeared to improve as $h^{+}$approached 5, whereas the skin-friction coefficient was overestimated indicatively when $h^{+}>7$. It should be emphasized here that these indications on the quality of the GLOFSFE estimations based on $h^{+}$were derived from the present observations. Since the aforementioned values of $h^{+}$may depend on the offset error in the oil-film thickness estimation, the indications may not be generalizable to other applications.

A representative, conservative estimation of the uncertainty in the laminar skin-friction coefficient determined from the GLOF data was $\Delta C_{f x}=1.5 \cdot 10^{-4}$. Compressible boundary-layer calculations were also performed using a laminar boundary-layer solver to obtain reference skinfriction data in the laminar-flow regions. With consideration of the aforementioned measurement uncertainty, the GLOFSFE estimations and the numerical results were found to be in reasonable agreement in portions of the chord for some of the examined data points. The stream-wise extent of the regions of reasonable agreement was significant at AoA $=1.8^{\circ}$ and $2.0^{\circ}$, but it reduced at lower angles of attack.

The quantitative GLOFSFE estimations in the laminarflow regions also enabled the quantitative identification of the location of transition onset with a maximal uncertainty of $5 \%$ of the chord (generally $3 \%$ or less). Considering the experimental uncertainties, the locations of transition onset estimated via GLOFSFE were generally in agreement with the transition locations detected via TSP in another experiment (Costantini et al. 2020), which was conducted for the same test cases as those examined in the present work. The transition location was observed to move upstream when the angle of attack was increased from AoA $=-0.4^{\circ}$ to $0.8^{\circ}$, as induced by the more pronounced pressure minimum and by the following stronger adverse pressure gradient. At AoA $>0.8^{\circ}$, the transition location was seen to move downstream with increasing angle of attack; this evolution was due to the change in the pressure distribution at such transonic flow conditions. Moreover, at $0.8^{\circ} \leq$ AoA $\leq 1.8^{\circ}$, the estimated location of transition onset was found to be close to the predicted location of laminar separation, in agreement with the expectation of transition starting shortly downstream of boundary-layer separation induced by a strong adverse pressure gradient.

The integration of the optical and conventional measurement techniques enabled the evaluation of the global influence of the oil-film setup on the aerodynamic coefficients. The lift and pitching moment coefficients, estimated after integration of the surface pressure distributions measured aside of the base coat, were generally unaffected by the oilfilm setup. However, the presence of the base coat already led to a significant increase in the drag coefficient (estimated 
via wake-deficit integration) for most of the examined data points, as compared to the clean configuration. Very probably, this was mainly due to the turbulent wedges originating from the base-coat edges. The additional drag increases in the presence of the oil film were negligible or small when the oil film was thin, whereas the larger additional $C_{D}$ increases were induced by additional turbulent wedges occurring in the oil-film area.

Based on the challenges encountered in the present work, the following improvements are recommended for future GLOF measurements in transonic wind-tunnel experiments at high Reynolds numbers:

- The base coat should be considered for the prevention of the offset intensity error and for the elimination of the span-wise discontinuities at the base-coat edges, which would otherwise induce turbulent wedges. An ideal surface would be a mirror-finished metallic surface that cannot absorb the luminescent dye. In this case, the excitation light reflection should be controlled so as not to be directed toward the camera.

If the oil film has to be applied onto a model surface that cannot be modified, and especially when the investigated model surface is already instrumented with pressure taps or other sensors, a thin, reflective metallic coat would be the most appropriate choice as a base coat.

- The oil-film thickness should be adjusted to a level such that the minimum thickness would be below the critical limit of roughness in both laminar- and turbulent-flow regions, while at the same time, the signal-to-noise ratio would remain sufficiently high. In the case of a still present offset intensity error, the measurement feasibility should be determined by the ratio of the oil luminescent intensity to the offset intensity.

According to the present observations and to the finding of Lee et al. (2020c), the optimal oil-film thickness in wall units $h^{+}$should be indicatively slightly below 5 . Note here that the coincidence of the recommendations about the optimal $h^{+}$for laminar- and turbulent-flow regions is probably incidental, since the oil-film thickness estimation in the laminar-flow regions examined in the present work was affected by an offset error. Nevertheless, even with consideration of the offset error, the oil-film thickness should be generally kept below $h^{+} \sim 5$ in order to avoid the risk of overestimating the skin friction.

Obviously, it is very challenging to guarantee the recommended $h^{+}$condition simultaneously in both laminar- and turbulent-flow regions. Therefore, the recommended oil-film thickness may be achieved, during the same wind-tunnel operation, first in the laminar boundary layer, and then - after running the wind tunnel for a long time-in the turbulent boundary layer. Alternatively, the oil-film thickness may be optimized for laminar- and turbulent-flow regions in different wind-tunnel runs.

- Efforts in the development of the oil-dye mixture should aim to the achievement of a brighter oil film. This would improve the signal-to-noise ratio of GLOF data, especially in measurements with a thin oil film.

- To improve repeatability, the measurements should indicatively focus on the 3-5 data points recorded after the first 3-4 different angles of attack, independently of the initial position and distribution of the oil film. In fact, the present observations showed that the first data points were still significantly affected by the starting oil conditions, whereas the signal-to-noise ratio was significantly reduced in the late data points.

- If laminar separation bubbles are expected to occur for a certain range of AoA, it is recommended to start the acquisitions from the angle of attack at which flow separation and reattachment are expected at the most downstream locations, and then increase/decrease the angle of attack to induce a progressive, upstream shift of the position of the laminar separation bubble. In this manner, the "imprinting" of regions of very low oil-film thickness in the subsequent data points should be avoided.

- A reduction of the oil viscosity, as compared to that considered in this work, would be a major advantage for future GLOF measurements with a thin oil film. However, it should be accepted that the oil would relatively quickly reach the trailing edge and then separate from the model, thus possibly damaging the wake-rake probes and contaminating the wind tunnel.

A global skin-friction estimation without appreciable flow disturbance and with higher accuracy is expected to be achievable with these improvements.

Acknowledgements The present study was supported in part by JSPS KAKENHI Grant Number $19 H 00800$ and JST Presto Grant Number JPMJPR1678. The experiments were conducted within the framework of the DLR VicToria Project, which provided financial and organizational support for this research. The authors are also thankful to: C. Fuchs (DLR) for the support in the model assembly, in the preparation of the Scheimpflug adapter, for the application of the base coat and for the polishing of the model leading edge region; T. Kleindienst (DLR) for the support in the installation of the measurement setup; U. Henne (DLR) for the assistance in the preparation of the data acquisition system; J. Agocs (DLR) for the support in the preparation of the Scheimpflug adapter and for the photographs of the experimental setup; A. Benkel (DNW) for the support during the test campaign and for the assistance in the evaluation of the uncertainties in the wind-tunnel parameters; M. Aschoff, M. Bruse, S. Fiedler, A. Grimme, B. Henne, K. Huber, M. Jacobs, N. Kretschmer, R. Lesjak, M. Löhr, M. Mikusch, K. Steiner, H. Uhlemann, and I. Volkmann-Steins (DNW) for the support during the test campaign; T. Gleisberg and M. Tegeler (DLR) for the construction of the investigated model insert; S.-M. Beyer, K. Ehbrecht, B. Eilerts, and S. Reinelt for the manufacturing of the model insert, for its instrumentation and for its installation in the main part of the model; M. Kube, A. Kunis, P. Schlöder, and K. Thüne 
(DLR) for the model installation in the DNW-TWG test section; and K. Borchert (DLR) as well as L. Schuster and H. Rosemann (formerly DLR) for the helpful indications on the VA-2 model. The anonymous reviewers are also acknowledged for their valuable comments, which helped the authors to improve the quality of the manuscript.

Funding Open Access funding enabled and organized by Projekt DEAL.. Open Access funding enabled and organized by Projekt DEAL.

Open Access This article is licensed under a Creative Commons Attribution 4.0 International License, which permits use, sharing, adaptation, distribution and reproduction in any medium or format, as long as you give appropriate credit to the original author(s) and the source, provide a link to the Creative Commons licence, and indicate if changes were made. The images or other third party material in this article are included in the article's Creative Commons licence, unless indicated otherwise in a credit line to the material. If material is not included in the article's Creative Commons licence and your intended use is not permitted by statutory regulation or exceeds the permitted use, you will need to obtain permission directly from the copyright holder. To view a copy of this licence, visit http://creativecommons.org/licenses/by/4.0/.

\section{References}

Amecke J (1986) Direct calculation of wall interferences and wall adaptation for two-dimensional flow in wind tunnels with closed walls. Tech. Rep. TM 88523, NASA, Washington, DC

Barlow JB, Rae WH, Pope A (1999) Low-Speed Wind Tunnel Testing. Aerospace engineering, mechanical engineering, Wiley

Binder B, Riethmueller L, Tusche S, Wulf R (1992) Die Modernisierung des Transsonischen Windkanals in Göttingen. Tech. Rep. DGLR-92-03-071, DGLR-Jahrestagung 1992, Bremen, Germany

Björck Å (1996) Numerical methods for least squares problems, vol 51. Society for Industrial and Applied Mathematics, Philadelphia

Blasius H (1908) Grenzschichten in Flüssigkeiten mit kleiner Reibung. Zeitschrift für Mathematik und Physik 56(1):1-37

Bohning R, Zierep J (1986) Calculation of 2d turbulent shock/boundary-layer interaction at curved surfaces with suction and blowing. In: Turbulent Shear-Layer/Shock-Wave Interactions, Springer, pp $105-112$

Braslow AL (1966) A review of factors affecting boundary-layer transition. Tech. Rep. TN-D-3579, NASA, Hampton, VA, https://ntrs. nasa.gov/search.jsp?R=19660023650

Braslow AL, Hicks RM, Harris RV (1966) Use of grit-type boundary-layer-transition trips on wind-tunnel models. Tech. Rep. TN-D-3579, NASA, Washington, DC, https://ntrs.nasa.gov/searc h.jsp?R=19660026829

Brown JL, Naughton JW (1999) The thin oil film equation. Tech. Rep. NASA/TM-1999-208767, NASA, Washington, DC, https://ntrs. nasa.gov/search.jsp?R=19990047906

Costantini M (2016) Experimental analysis of geometric, pressure gradient and surface temperature effects on boundary-layer transition in compressible high Reynolds number flow. PhD thesis, RWTH Aachen

Costantini M, Risius S, Koch S, Fuchs C, Gerhard U, Hein S, Klein C (2019) Experimental study of bump effects on boundary-layer transition in compressible high Reynolds number flow. Exp Thermal Fluid Sci 106:234-254. https://doi.org/10.1016/j.expthermfl usci.2019.04.027

Costantini M, Miozzi M, Henne U, Klein C (2020) Transition study on supercritical airfoil in compressible high-Reynolds number flow via Temperature-Sensitive Paint. In: 25th International Congress of Theoretical and Applied Mechanics (ICTAM2020+1), IUTAM, Accepted for presentation, pp 1-2

Courant R, Friedrichs K, Lewy H (1967) On the partial difference equations of mathematical physics. IBM J Res Dev 11(2):215234. https://doi.org/10.1147/rd.112.0215

Driver DM (2003) Application of oil-film interferometry skin-friction measurement to large wind tunnels. Exp Fluids 34(6):717-725

Heikkila J (2000) Geometric camera calibration using circular control points. IEEE Trans Pattern Anal Mach Intell 22(10):1066-1077. https://doi.org/10.1109/34.879788

Holmes BJ, Obara CJ, Martin GL, Domack CS (1985) Manufacturing tolerances for natural laminar flow airframe surfaces. SAE Transactions pp 522-531

Husen NM, Woodiga S, Liu T, Sullivan JP (2014) Global luminescent oil-film skin-friction meter generalized to three-dimensional geometry and applied to FAITH Hill. In: 52nd AIAA Aerospace Sciences Meeting, p 8. https://doi.org/10.2514/6.2014-1237

Husen NM, Roozeboom N, Liu T, Sullivan JP (2015) Global skinfriction measurements using particle image surface flow visualization and a luminescent oil-film. In: 53rd AIAA Aerospace Sciences Meeting, p 10. https://doi.org/10.2514/6.2015-0022

Husen NM, Liu T, Sullivan JP (2018) The ratioed image film thickness meter. Meas Sci Technol 29(6):065301. https://doi. org/10.1088/1361-6501/aabd27

Krenkel L (2012) Untersuchung des Einflusses von Wirbelgeneratoren auf die Stoß-/Grenzschicht-Wechselwirkung. PhD thesis, RWTH Aachen

Krogmann P, Stanewsky E, Thiede P (1984) Transonic shock/boundary layer interaction control. In: Proceedings of the 14th Congress of ICAS, vol 84, pp 297-307

Lee C, Lee T, Nonomura T, Asai K (2020a) Evaluating the applicability of a phase-averaged processing of skin-friction field measurement using an optical flow method. J Vis. https://doi. org/10.1007/s12650-020-00667-6

Lee T, Liu T (2018) Global luminescent oil film image based skin friction field estimator. Available online, https://github.com/ technolojin/GLOFSFE

Lee T, Nonomura T, Asai K, Liu T (2018) Linear least-squares method for global luminescent oil film skin friction field analysis. Rev Sci Instrum 89(6):065106. https://doi. org/10.1063/1.5001388

Lee T, Lee C, Nonomura T, Asai K (2020b) Unsteady skin-friction field estimation based on global luminescent oil-film image analysis. J Vis https://doi.org/10.1007/s12650-020-00661-y

Lee T, Nonomura T, Asai K, Naughton JW (2020c) Validation and uncertainty analysis of global luminescent oil-film skin-friction field measurement. Meas Sci Technol 31(3):035204. https://doi. org/10.1088/1361-6501/ab512a

Leuckert J (2012) Vergleichende Untersuchungen über die Eignung flächiger Sensorarrays zur Charakterisierung instationärer Strömungsphänomene in kompressiblen Strömungen. $\mathrm{PhD}$ thesis, TU Berlin

Liu T (2019) Global skin friction measurements and interpretation. Prog Aerosp Sci. https://doi.org/10.1016/j.paerosci.2019.100584

Liu T, Sullivan JP (1998) Luminescent oil-film skin-friction meter. AIAA J 36(8):1460-1465. https://doi.org/10.2514/2.538

Liu T, Woodiga S (2011) Feasibility of global skin friction diagnostics using temperature sensitive paint. Meas Sci Technol 22(11):115402. https://doi.org/10.1088/0957-0233/22/11/115402

Liu T, Cattafesta LN, Radeztsky RH, Burner AW (2000) Photogrammetry applied to wind-tunnel testing. AIAA J 38(6):964-971. https://doi.org/10.2514/2.1079

Liu T, Montefort J, Woodiga S, Merati P, Shen L (2008) Global luminescent oil-film skin-friction meter. AIAA J 46(2):476-485. https ://doi.org/10.2514/2.538 
Liu T, Woodiga S, Ma T (2011) Skin friction topology in a region enclosed by penetrable boundary. Exp Fluids 51(6):1549-1562. https://doi.org/10.1007/s00348-011-1171-6

Lunte J, Schülein E (2020) Wall shear stress measurements by whitelight oil-film interferometry. Exp Fluids 61(3):1-12. https://doi. org/10.1007/s00348-020-2917-9

Mateer GG, Seegmiller HL, Hand LA, Szodruch J (1992) An experimental investigation of a supercritical airfoil at transonic speeds. NASA Ames Research Center Technical Report (103933)

Mikhail EM, Bethel JS, McGlone CJ (2001) Introduction to modern photogrammetry. Wiley, New York

Miozzi M, Capone A, Costantini M, Fratto L, Klein C, Di Felice F (2019) Skin friction and coherent structures within a laminar separation bubble. Exp Fluids 60(1):13. https://doi.org/10.1007/ s00348-018-2651-8

Miozzi M, Di Felice F, Klein C, Costantini M (2020) Taylor hypothesis applied to direct measurement of skin friction using data from Temperature Sensitive Paint. Exp Thermal Fluid Sci. https://doi. org/10.1016/j.expthermflusci.2019.109913

Monson DJ, Mateer GG, Menter FR (1993) Boundary-layer transition and global skin friction measurement with an oil-fringe imaging technique. SAE Trans. https://doi.org/10.4271/932550

Mosharov V, Radchenko V, Klein C, Henne U, Kirmse T (2011) Evaluation of PISFV method in subsonic wind tunnel. In: Proceedings of 11th International Conference on Fluid Control, Measurements and Visualization (FLUCOME 2011), Keelung, Taiwan, 23

Mosharov VE, Orlov AA, Radchenko VN (2006) Application of correlation analysis in surface flow visualization with oil film. In: Dubnistchev YN, Rinkevichyus BS (eds) Optical methods of flow investigation, International Society for Optics and Photonics, SPIE, vol 6262, pp 82-89. https://doi.org/10.1117/12.683012

Naughton JW, Brown JL (1996) Surface interferometric skin-friction measurement technique. In: Advanced Measurement and Ground Testing Conference, p 2183. https://doi.org/10.2514/6.1996-2183

Naughton JW, Liu T (2007) Photogrammetry in oil-film interferometry. AIAA J 45(7):1620-1629. https://doi.org/10.2514/1.24634

Rosemann H, Stanewsky E, Hefer G (1995) The cryogenic ludwieg-tube of DLR and its new adaptive wall test section. In: 26th AIAA Fluid Dynamics Conference, p 9. https://doi. org/10.2514/6.1995-2198

Rubesin MW, Viegas JR (1989) Turbulence and modeling in transonic flow. In: Transonic symposium: theory, application, and experiment, NASA, Langley Research Center, vol 1, pp 981-610. https ://ntrs.nasa.gov/search.jsp?R=19890011580
Schlichting H (1968) Boundary-layer theory. McGraw Hill Book Co., New York. https://doi.org/10.1007/978-3-662-52919-5

Schrauf G (1998) COCO - A program to compute velocity and temperature profiles for local and nonlocal stability analysis of compressible, conical boundary layers with suction. ZARM Technik report

Schrauf G, von Geyr H (2020) Simplified Hybrid Laminar Flow Control for the A320 fin aerodynamic and system design. First results. In: AIAA Scitech 2020 Forum, p 13. https://doi. org/10.2514/6.2020-1536

Smith AMO, Clutter DW (1959) The smallest height of roughness capable of affecting boundary-layer transition. J Aerosp Sci 26(4):229-245

Streit T, Wedler S, Kruse M (2015) DLR natural and hybrid transonic laminar wing design incorporating new methodologies. Aeronaut J 119(1221):1303-1326. https://doi.org/10.1017/S000192400 0011283

Tanner LH, Blows LG (1976) A study of the motion of oil films on surfaces in air flow, with application to the measurement of skin friction. J Phys E: Sci Instrum 9(3):194. https://doi. org/10.1088/0022-3735/9/3/015

Thiede P, Krogmann P (1989) Passive control of transonic shock/ boundary layer interaction. In: Symposium Transsonicum III, Springer, pp 379-388. https://doi.org/10.1007/978-3-642-83584 $-1 \_30$

Thiede P, Krogmann P, Stanewsky E (1984) Active and passive shock/ boundary layer interaction control on supercritical airfoils. Tech. Rep. AGARD CP-365, AGARD, Brussels

Weiand P, Michelis S, Gardner AD (2017) Numerical simulation of an adaptive wall in a virtual transonic wind tunnel. AIAA J 55(9):3214-3218. https://doi.org/10.2514/1.J055481

Woodiga S, Liu T (2009) Skin friction fields on delta wings. Exp Fluids 47(6):897. https://doi.org/10.1007/s00348-009-0686-6

Zhong H, Woodiga S, Wang P, Shang J, Cui X, Wang J, Liu T (2015) Skin-friction topology of wing-body junction flows. Eur $\mathrm{J}$ Mech B/Fluids 53:55-67. https://doi.org/10.1016/j.euromechfl u.2015.04.002

Publisher's Note Springer Nature remains neutral with regard to jurisdictional claims in published maps and institutional affiliations. 